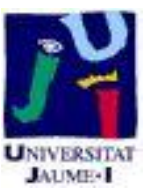

Título artículo / Títol article: Extrinsic Isoperimetric Analysis on Submanifolds with Curvatures Bounded from Below

Autores / Autors

Steen Markvorsen, Vicente Palmer

Revista:

Journal of Geometric Analysis

Versión / Versió:

Postprint del autor

Cita bibliográfica / Cita

MARKVORSEN, Steen; PALMER, Vicente.

bibliogràfica (ISO 690):

Extrinsic Isoperimetric Analysis on Submanifolds

with Curvatures Bounded from Below. Journal of

Geometric Analysis,

2010, vol. 20, no 2, p. 388-421.

url Repositori UJI:

http://hdl.handle.net/10234/70100 


\title{
EXTRINSIC ISOPERIMETRIC ANALYSIS ON SUBMANIFOLDS WITH CURVATURES BOUNDED FROM BELOW
}

\author{
STEEN MARKVORSEN\# AND VICENTE PALMER*
}

\begin{abstract}
We obtain upper bounds for the isoperimetric quotients of extrinsic balls of submanifolds in ambient spaces which have a lower bound on their radial sectional curvatures. The submanifolds are themselves only assumed to have lower bounds on the radial part of the mean curvature vector field and on the radial part of the intrinsic unit normals at the boundaries of the extrinsic spheres, respectively. In the same vein we also establish lower bounds on the mean exit time for Brownian motions in the extrinsic balls, i.e. lower bounds for the time it takes (on average) for Brownian particles to diffuse within the extrinsic ball from a given starting point before they hit the boundary of the extrinsic ball. In those cases, where we may extend our analysis to hold all the way to infinity, we apply a capacity comparison technique to obtain a sufficient condition for the submanifolds to be parabolic, i.e. a condition which will guarantee that any Brownian particle, which is free to move around in the whole submanifold, is bound to eventually revisit any given neighborhood of its starting point with probability 1 . The results of this paper are in a rough sense dual to similar results obtained previously by the present authors in complementary settings where we assume that the curvatures are bounded from above.
\end{abstract}

\section{INTRODUCTION}

Given a precompact domain $\Omega$ in a Riemannian manifold $M$, the isoperimetric quotient for $\Omega$ measures the ratio between the volume of the boundary and the volume of the enclosed domain: $\mathcal{Q}(\Omega)=$ $\operatorname{Vol}(\partial \Omega) / \operatorname{Vol}(\Omega)$. These volume measures and this quotient are descriptors of fundamental importance for obtaining geometric and analytic information about the manifold $M$. In fact, this holds true on every zoom level, be it global, local, or micro-local.

2000 Mathematics Subject Classification. Primary 53C42, 58J65, 35J25, $60 \mathrm{~J} 65$.

Key words and phrases. Submanifolds, extrinsic balls, radial convexity, radial tangency, mean exit time, isoperimetric inequalities, volume bounds, parabolicity.

\# Work partially supported by the Danish Natural Science Research Council and DGI grant MTM2004-06015-C02-02.

* Work partially supported by the Caixa Castelló Foundation, DGI grant MTM2004-06015-C02-02, and by the Danish Natural Science Research Council. 
1.1. On the global level. A classical quest is to find necessary and sufficient conditions for the type of a given manifold: Is it hyperbolic or parabolic? As already alluded to in the abstract, parabolicity is a first measure of the relative smallness of the boundary at infinity of the manifold: The Brownian particles are bound to eventually return to any given neighborhood of their starting point - as in $\mathbb{R}^{2}$; they do not get lost at infinity as they do in $\mathbb{R}^{3}$ (which the simplest example of a transient manifold).

In [14 T. Lyons and D. Sullivan collected and proved a number of equivalent conditions (the so-called Kelvin-Nevanlinna-Royden criteria) for non-parabolicity, i.e. hyperbolicity: The Riemannian manifold $(M, g)$ is hyperbolic if one (thence all) of the following equivalent conditions are satisfied: (a) $M$ has finite resistance to infinity, (b) $M$ has positive capacity, (c) $M$ admits a Green's function, (d) There exists a precompact open domain $\Omega$, such that the Brownian motion starting from $\Omega$ does not return to $\Omega$ with probability 1 , (e) $M$ admits a square integrable vector field with finite, but non-zero, absolute divergence. In particular the capacity condition (b) implies that $(M, g)$ is parabolic if it has vanishing capacity - a condition which we will apply in section 11

Returning now to the role of isoperimetric information: J. L. Fernandez showed in [3] that $M$ is hyperbolic if the so-called (rooted) isoperimetric profile function $\phi(t)$ has a quare integrable reciprocal, i.e. $\quad \int^{\operatorname{Vol}(M)} \phi^{-2}(t) d t<\infty$. Here the $\Omega_{0}$-rooted profile function is molded directly from isoperimetric information as follows:

$$
\begin{aligned}
\phi(t)=\inf \{ & \operatorname{Vol}(\partial \Omega) \mid \\
& \Omega \text { is a smooth relatively compact domain in } M, \\
& \left.\Omega \supset \Omega_{0}, \quad \text { and } \operatorname{Vol}(\Omega) \geq t\right\} .
\end{aligned}
$$

The volume of a non-parabolic manifold $M$ is necessarily infinite. In fact, a finite volume manifold is parabolic by the following theorem due to Grigor'yan, Karp, Lyons and Sullivan, and Varopoulos. See [7] section 7.2 for an account of this type of results, which again is stated in terms of the simplest possible 'isoperimetric' information: Let $B_{r}(q)$ denote the geodesic ball centered at $q$ in $M$ and with radius $r$. If there exists a point $q$ such that one (or both) of the following conditions is satisfied

$$
\begin{gathered}
\int^{\infty} \frac{r}{\operatorname{Vol}\left(B_{r}(q)\right)} d r=\infty \\
\int^{\infty} \frac{1}{\operatorname{Vol}\left(\partial B_{r}(q)\right)} d r=\infty
\end{gathered}
$$

then $M$ is parabolic.

In the present paper we obtain generalizations of this parabolicity condition. They are obtained for submanifolds in ambient spaces with a 
lower bound on curvatures by using a capacity comparison technique in combination with the Kelvin-Nevanlinna-Royden condition (b) as stated above, see Proposition 10.4 and Theorem 10.5. These results complement - and are in a rough sense dual to - previous hyperbolicity results that we have obtained using a corresponding upper bound on the curvatures of the ambient spaces, see [19, 20].

1.2. On the local level. If the boundary of a given domain is relatively small as compared to the domain itself, we also expect the mean exit time for Brownian motion to be correspondingly larger. The main concern of the present paper is to show an upper bound on the isoperimetric quotients of the so-called extrinsic balls of submanifolds under the essential assumption that the ambient spaces have a lower bound on their curvatures, see Theorem 7.1. The result for the mean exit time (from such extrinsic balls) then follows, as observed and proved in Theorem 10.2. These results are again dual to results which have been previously obtained under the condition of an upper bound on the curvature of the ambient space, see [25, 18] and [15, 24], respectively.

1.3. On the micro-local level. When considering again the intrinsic geodesic balls $B_{\varepsilon}(q)$ of $M$ centered at a fixed point $q$ and assuming that the radius $\varepsilon$ is approaching 0 , then the Taylor series expansion of the volume function of the corresponding metric ball (or metric sphere) contains information about the curvatures of $M$ at $q$ - a classical result (for surfaces) obtained by Gauss and developed by A. Gray and L. Vanhecke in their seminal work [5]. Moreover, the geodesic metric balls have been analyzed by A. Gray and M. Pinsky in [4] in order to extract the geometric information contained in that particular function of $\varepsilon$, which gives the mean exit time from the center point $q$ of the metric ball $B_{\varepsilon}(q)$, see Theorem 10.1 below in section 10 .

To motivate even further the extrinsic geometric setting under consideration in the present paper, we mention here also yet another nice observation due to L. Karp and M. Pinsky concerning submanifolds in $\mathbb{R}^{n}$; see [12, 11], where they show how to extract combinations of the principal curvatures of a submanifold at a point $q$ from suitable power series expansions of the respective functions $\operatorname{Vol}\left(D_{\varepsilon}(q)\right), \operatorname{Vol}\left(\partial D_{\varepsilon}(q)\right)$, and $E_{D_{\varepsilon}(q)}$, where $D_{\varepsilon}(q)$ is the extrinsic ball of (extrinsic) radius $\varepsilon$ centered at $q$, and $E$ is the mean exit time function.

On all levels then, be it global, local, or micro-local, as well as from both viewpoints, intrinsic or extrinsic, we thus encounter a fundamental interplay and inter-dependence between the highly instrumental geometric concepts of measure, shape, and diffusion considered here, namely the notions of volume, curvature, and exit time, respectively. 
1.4. Outline of the paper. In the first three sections 2 , 3, and 4 , we first provide intuitive versions, shadows, of our main results, i.e. we present the general results under stronger conditions than actually needed and compare them in particular with previous techniques for obtaining parabolicity (for surfaces of revolution) due to J. Milnor and K. Ichihara. In section 5 we then begin to establish the technical machinery for the paper and give precise definitions of the geometric bounds needed as preparation for our definition of what we call an Isoperimetric Constellation in section 6. This key notion is then applied in section 7 to present and prove our main isoperimetric result, Theorem 7.1. Various consequences of the main result and its proof are shown in sections 8 and 9 . In particular we find new inequalities involving the volumes of extrinsic balls and their derivatives as well as intrinsic versions of our main results. In section 10 we find a lower bound on the mean exit time function from extrinsic balls, and establish in section 11 an inequality for the capacities of extrinsic annular domains, which is then finally applied to prove the parabolicity result, Theorem 10.5, as alluded to above.

\section{A first Glimpse of the Main Results}

We first facilitate intuition concerning our main results by considering some of their consequences for submanifolds in constant curvature ambient spaces - in particular for surfaces in $\mathbb{R}^{3}$. This seems quite relevant and worthwhile, because even in these strongly restricted settings we find results, which we believe are of independent interest. The results presented here are but shadows of the general results. The full versions of the main theorems appear in the sections below as indicated in the Outline, section 1.4 .

2.1. Strong Assumptions and Constant Curvature. The general strong conditions applied for these initial statements are as follows: We let $P^{m}$ denote a complete immersed submanifold in an ambient space form $N^{n}=\mathbb{K}_{b}^{n}$ with constant sectional curvature $b \leq 0$. Suppose further that $P$ is radially mean $C$-convex in $N$ as viewed from a point $p$ in the following sense: The unique oriented, arc length parametrized geodesic $\gamma_{p \rightarrow x}$ from $p$ to $x \in P$ in the ambient space $\mathbb{K}_{b}^{n}$ has an inner product with the mean curvature vector $H_{P}(x)$ of $P$ in $N$ at $x$, which is bounded as follows:

$$
\mathcal{C}(x)=-\left\langle\gamma_{p \rightarrow x}^{\prime}, H_{P}(x)\right\rangle \geq C
$$

for some constant $C$. This condition with $C=0$ is e.g. satisfied by convex hypersurfaces, see remark 5.2, as well as by all minimal submanifolds. We then consider a special type of compact subsets of $P$, the so-called extrinsic balls $D_{R}$, which for any given $R>0$ consists of those points $x$ in $P$, which have extrinsic distance to $p$ less than or 

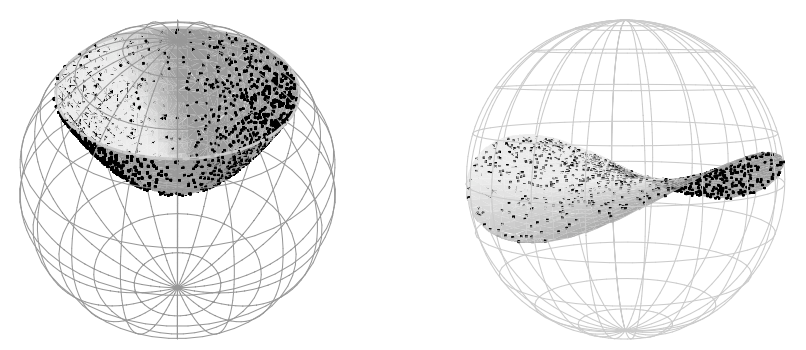

Figure 1. Examples of extrinsic discs cut by a sphere in $\mathbb{R}^{3}$ from a paraboloid and from a minimal surface, respectively. Both extrinsic discs are radially mean $0-$ convex from the center point of the cutting sphere. Theorem $\mathrm{A}$ provides an upper bound on the isoperimetric quotients for the respective extrinsic discs, cf. remark 2.1.

equal to $R$, see Figure 1.

At each point $x$ on its boundary $\partial D_{R}$, the extrinsic ball $D_{R}$ has a unique outward pointing unit vector $\nu_{x}$, which has the following inner product with the unit tangent vector of $\gamma_{p \rightarrow x}$ :

$$
\mathcal{T}(x)=\left\langle\gamma_{p \rightarrow x}^{\prime}, \nu_{x}\right\rangle .
$$

We let $g(R)$ denote the minimum tangency value $\mathcal{T}$ along the boundary of the extrinsic ball $D_{R}$ - in general:

$$
g(r)=\min _{x \in \partial D_{r}} \mathcal{T}(x)
$$

An instrumental assumption to be satisfied throughout this paper, is that $g(r)>0$ for all $r \leq R$ for each $D_{R}$ under consideration. In particular this then implies that the extrinsic balls always have only one connected compact component because any other compact component than the ones containing $p$ would automatically have a point $q$ at minimum distance to $p$, where then $\mathcal{T}(q)=0$, which contradicts $g(r(q))>0$.

With these initial ingredients and concepts we can now state the following first instances of our main theorems:

Theorem A (The full version is stated in Theorem 7.1). If we assume the strong conditions stated above in section 2.1, then the following upper bound on the isoperimetric quotients for extrinsic balls holds true for $C \leq \sqrt{-b}$ :

$$
\frac{\operatorname{Vol}\left(\partial D_{R}\right)}{\operatorname{Vol}\left(D_{R}\right)} \leq \frac{m}{g(R)}\left(\eta_{Q_{b}}(R)-C\right)
$$


where $\eta_{Q_{b}}(R)$ is the constant mean curvature of the metric hyper-sphere of radius $R$ in the space form of constant curvature $b$ (see (5.9)); this function has $\lim _{r \rightarrow \infty} \eta_{Q_{b}}(r)=\sqrt{-b}$.

Remark 2.1. For the surfaces displayed in Figure 1 the isoperimetric inequality reads as follows (since $C=0$ and $b=0$ ):

$$
\frac{\operatorname{Length}\left(\partial D_{R}\right)}{\operatorname{Area}\left(D_{R}\right)} \leq \frac{2}{R g(R)},
$$

where $g(R)$ is the lower bound of the tangency function $\mathcal{T}$ along the boundary curve $\partial D_{R}$ of the extrinsic disc, see (2.2) and (2.3).

Theorem B (The full version is stated in Theorem 10.2). We assume the strong general conditions stated above in section [2.1. Then the mean exit time $E_{R}(x)$, i.e. the time that it takes (on average) for a Brownian particle starting from a point $x \in D_{R}$ to reach the boundary $\partial D_{R}$ enjoys a lower bound of the following radial type:

$$
E_{R}(x) \geq \mathcal{E}_{R}(r(x)),
$$

where $\mathcal{E}_{R}(r)$ is a well defined radial function, which only depends on $R$, $b, C$, and $g(r)$. The precise form of this dependence will, of course, be substantially explicated below, see section 10 and Theorem 10.2 therein.

Theorem $\mathbf{C}$ (The full version is stated in Theorem 10.5). We assume the strong general conditions stated above in section 2.1 for all radii $R$ of the extrinsic disc $D_{R}$. Suppose further that

$$
\int^{\infty} Q_{b}(r) \exp \left(-\int_{1}^{r} \frac{m}{g^{2}(t)}\left(\eta_{Q_{b}}(t)-C\right) d t\right) d r=\infty \quad .
$$

Then $P^{m}$ is parabolic.

In particular, we may, and do, extract the following first corollaries directly from the above theorem.

Corollary 2.2. Let $P^{2}$ denote a two-dimensional radially mean 0convex surface in Euclidean space $\mathbb{R}^{n}$ with a radial tangency bounding function $g(r)$ which satisfies the following inequality for all sufficiently large $r$ :

$$
g(r) \geq \tilde{g}(r)=\sqrt{\frac{2 \log (r)}{1+2 \log (r)}} .
$$

Then $P^{2}$ is parabolic.

Remark 2.3. This particular result should be viewed in the light of the fact that there are well known minimal (hence 0-convex) surfaces in $\mathbb{R}^{3}$ - like Scherk's doubly periodic minimal surface - which are nonparabolic (i.e. hyperbolic), but which nevertheless also - in partial contrast to what could be expected from the above Corollary - support 
radial tangency functions $\mathcal{T}(x)$ which are 'mostly' close to 1 at infinity. The Scherk surface alluded to is the graph surface of the function $f(x, y)=\log (\cos (y) / \cos (x))$, which is smooth and well-defined on a checkerboard pattern in the $(x, y)$-plane. It was proved in [17 - via methods quite different from those considered in the present paper that Scherk's surface is hyperbolic. Roughly speaking, the Scherk's surface may be considered as the most 'slim' known hyperbolic surface in $\mathbb{R}^{3}$. If that surface is viewed from a point far away from the $(x, y)$-plane, the surface looks like two sets of parallel half-planes, both orthogonal to the $(x, y)$-plane, one set below and the other above, and the two sets being rotated $\pi / 2$ with respect to each other, see e.g. [16, pp. 46-49].

The radial tangency (from any fixed point $p$ in the $(x, y)$-plane) is 'mostly' close to 1 at infinity except for the points in the $(x, y)$-plane itself, where the tangency function is 'wiggling' sufficiently close to 0 , so that the condition 2.8 cannot be satisfied. In fact, if we accept for a moment the rough description of the surface as two sets of parallel half planes, then the integral over an extrinsic disc $D_{R}$ of the tangency function $\mathcal{T}(x)$ from $p=(0,0,0)$ is roughly $0.8 \operatorname{Area}\left(D_{R}\right)$ for large values of $R$. Note that for this simplified calculation the extrinsic disc consists of a finite number of flat half-discs, each one of which has radius $\sqrt{R^{2}-\rho_{0}^{2}}$, where $\rho_{0} \leq R$ denotes the orthogonal distance to $p$ from the plane containing the half-disc.

We have discussed this particular example at some length, because it seems to be a good example for displaying in purely geometric terms what goes on at or close to the otherwise still quite unknown borderline between hyperbolic and parabolic surfaces in $\mathbb{R}^{3}$. In other words, the tangency function $\mathcal{T}$ introduced here seems to have an interesting and instrumental rôle to play concerning the quest of finding a necessary and sufficient condition for a surface to be hyperbolic, resp. parabolic.

Corollary 2.4. Let $P^{m}$ denote an $m$-dimensional radially mean $C-$ convex submanifold in the space form $\mathbb{K}_{b}^{n}$ of constant curvature $b<0$. Suppose

$$
0 \leq m(\sqrt{-b}-C) \leq \sqrt{-b},
$$

and suppose that $P^{m}$ admits a radial tangency bounding function $g(r)$ which satisfies $g(r) \geq \tilde{g}(r)$ for all sufficiently large $r$, where now using shorthand notation $\tau=r \sqrt{-b}$ :

$$
\tilde{g}(r)=\sqrt{\frac{m r \log (r)(\sqrt{-b} \cosh (\tau)-C \sinh (\tau))}{\sinh (\tau) \log (r)+\tau \cosh (\tau) \log (r)+\sinh (\tau)}} .
$$

Then $P^{m}$ is parabolic.

Remark 2.5. This result should likewise be compared with the fact established in [20], that every minimal submanifold of any co-dimension 
in a negatively curved space form is transient. Such submanifolds are not $C$-convex, of course, for any positive $C$ (note that 2.9 implies $C>0$ ). We observe that the bounding function $\tilde{g}(r) \rightarrow 0$ for $r \rightarrow 0$ precisely when $C=\sqrt{-b}$. Therefore, in relation to the discussion in the previous remark 2.3, what 'induces' parabolicity in negatively curved ambient spaces is to a large extent the radial mean $C$-convexity assumption - not just the tangency condition $\mathcal{T}(x) \geq \tilde{g}(r(x))$.

Proof of Corollary 2.2. With $b=0$ we have $Q_{b}(r)=r$ and since $\tilde{g}(r)$ is designed to satisfy

$$
\frac{2}{r \tilde{g}^{2}(r)}=\frac{2 r \log (r)+r}{r^{2} \log (r)}=\frac{d}{d r} \log \left(r^{2} \log (r)\right)
$$

for sufficiently large values of $r$, say $r \geq A$, we get for some positive constant $c_{1}$ :

$$
-\int_{A}^{r} \frac{2}{t \tilde{g}^{2}(t)} d t=-\log \left(r^{2} \log (r)\right)+c_{1}
$$

so that, for some other positive constant $c_{2}$ :

$$
\begin{aligned}
& \int^{\infty} Q_{b}(r) \exp \left(-\int_{A}^{r} \frac{m \eta_{Q_{b}}(t)}{g^{2}(t)} d t\right) d r \\
& \geq \int^{\infty} r c_{2} \exp \left(-\int_{A}^{r} \frac{2}{t \tilde{g}^{2}(t)} d t\right) d r \\
& =\int^{\infty} \frac{c_{2}}{r \log (r)} d r \\
& =\infty,
\end{aligned}
$$

which then implies parabolicity according to theorem $\mathrm{C}$.

The proof of Corollary 2.4 follows essentially verbatim, except for handling the allowed $C$-interval for given $b$ and $m$. The condition (2.9) simply stems from the two obvious conditions, that the square root defining $\tilde{g}(r)$ in 2.10$)$ must be well-defined and less than 1 . It is of independent interest to note as well, that when $b$ approaches 0 then $C$ must go to 0 , i.e. we are then back in the case of Corollary 2.2.

\section{Previous Results for Surfaces}

We show in the next section, that a number of surfaces, including the catenoid and the hyperboloid of one sheet, are parabolic using the condition established in Corollary 2.2. Parabolicity of those surfaces is known already from criteria due to by Milnor and Ichihara, which we briefly outline here for comparison with our Corollary 2.2 - again mainly in order to support and facilitate intuition before stating and proving the full versions of our main results. 
J. Milnor considered 2D warped products (see Definition 5.4 in section 5p with general metrics molded by a warping functions $w(r)$ on $r \in[0, \infty[$ as follows:

$$
d s^{2}=d r^{2}+w^{2}(r) d \theta .
$$

Theorem 3.1 (Milnor, [22]). If the Gaussian curvature function $K(s)$ considered as a function of the distance $s$ from the pole of a model surface given as in (3.1) satisfies

$$
K(s) \geq-\frac{1}{s^{2} \log (s)},
$$

then the surface is parabolic.

A condition for parabolicity in terms of the total curvature of a given surface (not necessarily a model surface of revolution) is established by K. Ichihara:

Theorem 3.2 (Ichihara, [8]). If a 2-dimensional manifold $M^{2}$ has finite total absolute Gaussian curvature, i.e.

$$
\int_{M^{2}}|K| d \mu<\infty
$$

then it is parabolic.

\section{Examples AND Benchmarking Surfaces of Revolution}

The examples we have in mind are classical but suitably modified to provide well defined (and simple) extrinsic balls (discs) to exemplify our analysis. We consider piecewise smooth radially mean $0-$ convex surfaces of revolution in $\mathbb{R}^{3}$ constructed as follows. In the $(x, z)$-plane we consider the profile generating curve consisting of a (possibly empty) line segment along the $x$-axis: from $(0,0)$ to $(a, 0)$ for some $a \in[0, \infty[$ together with a smooth curve $\Gamma(u)=(x(u), z(u)), u \in[0, c]$ for some $c \in] 0, \infty$ [ with $x(u)>0$ for all $u$ and $x(0)=a$. The corresponding surfaces of revolution then (possibly) have a flat (bottom) disc of radius $a$, see Figure 2 .

The center of this disc, i.e. the origin $p=(0,0,0)$, will serve as the point from which the surfaces under consideration will be $p$-radially mean 0 -convex as well as radially symmetric via the specific choices of generating functions $x(u)$ and $z(u)$.

The extrinsic radius of the defining cutting sphere centered at $p$ is chosen to be $R(c)>a$, so that the corresponding extrinsic disc containing $p$ consists of the flat bottom disc of radius $a$ together with the following non-vanishing part of the surface of revolution:

$\Omega_{c}: r(u, v)=(x(u) \cos (v), x(u) \sin (v), z(u)), u \in[0, c], v \in[-\pi, \pi[$. 

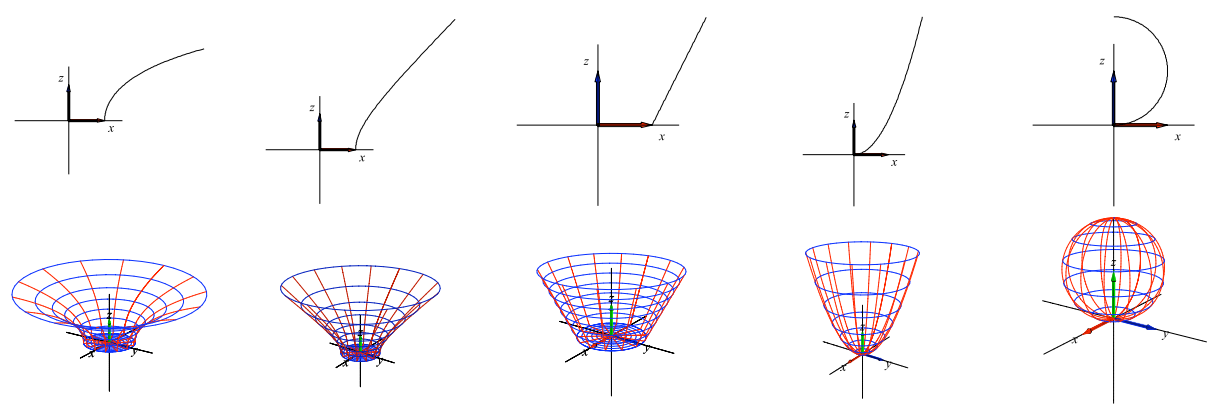

FiguRE 2. Generating profile curves and the corresponding surfaces of revolution. Counting from the left the surfaces are constructed from a Catenoid, Hyperboloid of one sheet, Cone, Paraboloid, and a Sphere, respectively. The three left-most surfaces are completed with a flat bottom disc as shown. These surfaces are all radially mean 0 -convex from the Origin. We illustrate in section 4 how the quite different criteria of Milnor, Ichihara, as well as the condition of Corollary 2.2 in the present paper show that all these surfaces are parabolic.

The area of this extrinsic disc is then a function of $a$ and $c$ as follows:

$$
\operatorname{Area}\left(D_{R(c)}\right)=A(a, c)=\pi a^{2}+2 \pi \int_{0}^{c} x(t) \sqrt{x^{\prime 2}(t)+z^{\prime 2}(t)} d t,
$$

and the length of its boundary is simply

$$
\operatorname{Length}\left(\partial D_{R(c)}\right)=L(a, c)=2 \pi x(c) .
$$

The exact isoperimetric quotient of the extrinsic disc of the resulting surface of revolution is thus

$$
\mathcal{Q}=\mathcal{Q}(a, c)=\frac{x(c)}{\left(a^{2} / 2\right)+\int_{0}^{c} x(t) \sqrt{x^{\prime 2}(t)+z^{\prime 2}(t)} d t}
$$

The main theorem of the present paper asserts that the quotient $\mathcal{Q}(a, c)$ is bounded from above by the right hand side of equation (2.5) - under the assumed condition, that the surface of revolution is radially mean 0-convex from $p$ :

$$
\frac{2}{R(c) g(R(c))}=\frac{2}{g(c) \sqrt{x^{2}(c)+z^{2}(c)}},
$$

where we use $g\left(u_{0}\right)$ as shorthand for $g\left(R\left(u_{0}\right)\right)$ which is the exact common radial tangency value for the surface of revolution at each point of the circle $\left(x\left(u_{0}\right) \cos (v), x\left(u_{0}\right) \sin (v), z\left(u_{0}\right)\right)$. It is by definition the inner 
product:

$$
\begin{aligned}
g\left(u_{0}\right) & =\left\langle\frac{\Gamma^{\prime}\left(u_{0}\right)}{\left\|\Gamma^{\prime}\left(u_{0}\right)\right\|}, \frac{\Gamma\left(u_{0}\right)}{\left\|\Gamma\left(u_{0}\right)\right\|}\right\rangle \\
& =\frac{x\left(u_{0}\right) x^{\prime}\left(u_{0}\right)+z\left(u_{0}\right) z^{\prime}\left(u_{0}\right)}{\sqrt{x^{\prime 2}\left(u_{0}\right)+z^{\prime 2}\left(u_{0}\right)} \sqrt{x^{2}\left(u_{0}\right)+z^{2}\left(u_{0}\right)}} .
\end{aligned}
$$

Therefore the comparison upper bound reduces to:

$$
\frac{2}{R(c) g(R(c))}=\frac{2 \sqrt{x^{\prime 2}(c)+z^{\prime 2}(c)}}{x(c) x^{\prime}(c)+z(c) z^{\prime}(c)} .
$$

The examples below then serve as illustrations of our main theorem within the category of surfaces of revolution. Given the functions $x(u)$ and $z(u)$ we simply verify the following inequality in each case:

$$
\mathcal{Q}(a, c)=\frac{A(a, c)}{L(a, c)} \leq \frac{2}{R(c) g(c)}
$$

which (as shown above) is equivalent to:

$$
\frac{x(c)}{\left(a^{2} / 2\right)+\int_{0}^{c} x(t) \sqrt{x^{\prime 2}(t)+z^{\prime 2}(t)} d t} \leq \frac{2 \sqrt{x^{\prime 2}(c)+z^{\prime 2}(c)}}{x(c) x^{\prime}(c)+z(c) z^{\prime}(c)}
$$

Simultaneously in our short presentations below we also illustrate how the conditions of Corollary 2.2, Theorems 3.1 and 3.2 apply as well to prove parabolicity of each one of the surfaces in question.

Example 4.1. The Catenoid is a minimal surface and hence radially mean 0-convex from any point on the surface; It is also clearly radially mean 0 -convex from $p$ in its truncated version considered here completed with a "flat disc bottom" of radius $a=1$. We have in this particular case:

$$
\begin{aligned}
& x(u)=\cosh (u) \\
& z(u)=u
\end{aligned}
$$

for $u \in[0, c]$. Our isoperimetric inequality (in the form of (4.6)) is easily verified for all $c$. The tangency function follows from equation (4.3) and is given by:

$$
\mathcal{T}(u, v)=g(u)=\frac{\sinh (u) \cosh (u)+u}{\cosh (u) \sqrt{\cosh (u)^{2}+u^{2}}},
$$

and the corresponding distance function to $p$, the origin, is:

$$
r(u, v)=\sqrt{\cosh (u)^{2}+u^{2}},
$$

It is a simple calculation to see that indeed the lower tangency bound of Corollary 2.2. (2.8), holds true, so that we can conclude parabolicity 
of the truncated catenoid. Moreover, the Gaussian curvature of the catenoid is:

$$
K(u, v)=-\frac{1}{\cosh ^{4}(u)}
$$

The surface integral of the absolute curvature is clearly finite. A direct calculation gives:

$$
\int_{M^{2}}|K| d \mu=2 \pi \int_{-\infty}^{\infty} \frac{1+\cosh (2 u)}{2 \cosh ^{4}(u)} d u=4 \pi .
$$

Icihara's condition in Theorem 3.2 then also gives parabolicity. To apply Milnor's condition, we calculate the arc length presentation:

$$
s(u)=\sinh (u),
$$

and parabolicity then follows from (3.2), which in this case reads:

$$
-\frac{1}{\cosh ^{4}(u)} \geq-\frac{1}{\sinh ^{2}(u) \log (\sinh (u))} .
$$

Example 4.2. The truncated and completed Hyperboloid of one sheet is also radially mean 0 -convex from the center point $p$ of its flat bottom, although this is not clear from its shape. Indeed, from the generating functions

$$
\begin{aligned}
& x(u)=\sqrt{1+u^{2}} \\
& z(u)=u
\end{aligned}
$$

a short calculation reveals the following non-negative radial mean convexity function - see also remark 4.3 :

$$
\mathcal{C}(x)=h(u(x))=\frac{u^{2}}{\left(2 u^{2}+1\right)^{5 / 2}} \geq 0 .
$$

The inequality (4.6) holds true for all $b \geq 0$ and our isoperimetric inequality is thus verified in this case. The curvature is

$$
K(u, v)=-\frac{1}{\left(1+2 u^{2}\right)^{2}}
$$

and the intrinsic distance is essentially:

$$
s(u)=i E(-u i, \sqrt{2}),
$$

where $E(z, k)$ is the incomplete elliptic integral of the second kind. Parabolicity of the standard hyperboloid of one sheet then follows from Milnor's condition:

$$
-\frac{1}{\left(1+2 u^{2}\right)^{2}} \geq-\frac{1}{s^{2}(u) \log (s(u))} .
$$

The surface integral of the absolute value of the Gauss curvature is again finite. A direct calculation gives:

$$
\int_{M^{2}}|K| d \mu=2 \pi \int_{-\infty}^{\infty} \frac{1}{\left(1+2 u^{2}\right)^{3 / 2}} d u=2 \pi \sqrt{2} .
$$


The tangency function is

$$
\mathcal{T}(u, v)=\frac{2 u \sqrt{1+u^{2}}}{1+2 u^{2}},
$$

and the corresponding extrinsic distance function to the origin is:

$$
r(u, v)=\sqrt{1+2 u^{2}} .
$$

It is a simple calculation to see that indeed the lower tangency bound of (2.8) holds true, so that parabolicity also stems from that condition.

Remark 4.3. Before considering the convex surfaces of revolution below - the paraboloids and spheres - we note in passing, that for surfaces of revolution of the type considered above, i.e. those with $z(u)=u$, we have the following differential inequality condition on $x(u)$, which is equivalent to radial mean 0 -convexity from $p$ for the corresponding surface of revolution, $\mathcal{C}(u) \geq 0$ :

$$
\left(x(u) x^{\prime \prime}(u)-x^{2}(u)-1\right)\left(x(u)-u x^{\prime}(u)\right) \geq 0 .
$$

In particular, for the hyperboloid, $x(u)=\sqrt{1+u^{2}}$, the left hand side of this inequality reads $2 u^{2} /\left(1+u^{2}\right)^{3 / 2}>0$. Moreover, we note that equality is obtained in the condition 4.22) (assuming $x(0)=1$ and $\left.x^{\prime}(0)=0\right)$ precisely when $x(u)=\cosh (u)$, i.e. for the catenoid.

Example 4.4. The truncated and completed Cone in Figure 2 is radially mean $0-$ convex from the point $p$. We have:

$$
\begin{aligned}
& x(u)=1+u \cos (\theta) \\
& z(u)=u \sin (\theta)
\end{aligned}
$$

for $u \in[0, c]$ and any constant $\theta \in[-\pi / 2, \pi / 2]$. The inequality (4.6) holds true for all $c \geq 0$ and all $\theta$, thus verifying our main isoperimetric inequality. The tangency function is:

$$
\mathcal{T}(u, v)=\frac{\cos (\theta)+u}{\sqrt{1+2 u \cos (\theta)+u^{2}}},
$$

and the corresponding distance function to the origin is:

$$
r(u, v)=\sqrt{1+2 u \cos (\theta)+u^{2}} .
$$

Again it is a simple calculation to see that the lower tangency bound of (2.8) holds true:

$$
\frac{\cos (\theta)+u}{\sqrt{1+2 u \cos (\theta)+u^{2}}} \geq \sqrt{\frac{\log \left(1+2 u \cos (\theta)+u^{2}\right)}{\log \left(1+2 u \cos (\theta)+u^{2}\right)+1}},
$$

so that parabolicity follows from our Corollary 2.2. Milnor's criterion is clearly satisfied as well since the Gaussian curvature is non-negative. 
Also, the (truncated) cones clearly have finite total absolute curvature, so Ichihara's criterion applies immediately as well.

Example 4.5. The Paraboloid is clearly convex and hence radially mean 0 -convex from the top point $p$ :

$$
\begin{aligned}
& x(u)=u \\
& z(u)=\alpha u^{2}
\end{aligned}
$$

for $u \in[0, c]$ and any constant $\alpha \in \mathbb{R}$. The inequality (4.6) holds true for all $c \geq 0$ and all $\alpha$. The tangency function is:

$$
\mathcal{T}(u, v)=\frac{1+2 \alpha^{2} u^{2}}{\sqrt{4 \alpha^{2} u^{2}+1} \sqrt{1+\alpha^{2} u^{2}}},
$$

and the since corresponding distance function to the origin is

$$
r(u, v)=u \sqrt{1+\alpha^{2} u^{2}},
$$

it is a simple calculation to see that indeed the lower tangency bound of (2.8) holds true, so that parabolicity follows.

Example 4.6. The round Sphere is clearly mean 0-convex from the south pole $p$; we have:

$$
\begin{aligned}
& x(u)=-\sin (u-\pi) \\
& z(u)=1+\cos (u-\pi)
\end{aligned}
$$

for $u \in[0, c], c \leq \pi$. In this interval the inequality 4.6 reduces to:

$$
\frac{\sin (c)}{1-\cos (c)} \leq \frac{2}{\sin (c)}
$$

which is clearly satisfied for all $c \in[0, \pi]$, so that we have again thereby verified our main isoperimetric inequality (2.4) in the reduced form of (2.5). Parabolicity follows in this (extreme) case from mere compactness of the complete surface of a sphere.

\section{Geometric Bounds from Below and Model Space Carriers}

5.1. Lower Mean Convexity Bounds. Given an immersed, complete $m$-dimensional submanifold $P^{m}$ in a complete Riemannian manifold $N^{n}$ with a pole $p$, we denote the distance function from $p$ in the ambient space $N^{n}$ by $r(x)=\operatorname{dist}_{N}(p, x)$ for all $x \in N$. Since $p$ is a pole there is - by definition - a unique geodesic from $x$ to $p$ which realizes the distance $r(x)$. We also denote by $r$ the restriction $\left.r\right|_{P}: P \longrightarrow \mathbb{R}_{+} \cup\{0\}$. This restriction is then called the extrinsic distance function from $p$ in $P^{m}$. The corresponding extrinsic metric balls of (sufficiently large) radius $R$ and center $p$ are denoted by $D_{R}(p) \subseteq P$ and defined as follows:

$$
D_{R}(p)=B_{R}(p) \cap P=\{x \in P \mid r(x)<R\},
$$


where $B_{R}(p)$ denotes the geodesic $R$-ball around the pole $p$ in $N^{n}$. The extrinsic ball $D_{R}(p)$ is assumed throughout to be a connected, pre-compact domain in $P^{m}$ which contains the pole $p$ of the ambient space. Since $P^{m}$ is (unless the contrary is clearly stated) assumed to be unbounded in $N$ we have for every sufficiently large $R$ that $B_{R}(p) \cap P \neq$ $P$.

In order to control the mean curvatures $H_{P}(x)$ of $P^{m}$ at distance $r$ from $p$ in $N^{n}$ we introduce the following definition:

Definition 5.1. The $p$-radial mean curvature function for $P$ in $N$ is defined in terms of the inner product of $H_{P}$ with the $N$-gradient of the distance function $r(x)$ as follows:

$$
\mathcal{C}(x)=-\left\langle\nabla^{N} r(x), H_{P}(x)\right\rangle \text { for all } x \in P .
$$

We say that the submanifold $P$ satisfies a radial mean convexity condition from $p \in P$ when we have a smooth function $h: P \mapsto \mathbb{R}$, such that

$$
\mathcal{C}(x) \geq h(r(x)) \text { for all } \quad x \in P .
$$

The submanifold $P$ is called radially mean $C$-convex when its $p$-radial mean curvature is bounded from below by the constant $C$. Minimal submanifolds and convex hypersurfaces are radially mean 0 -convex, cf. the following remark.

Remark 5.2. A hypersurface $P^{n-1}$ in $\mathbb{R}^{n}$ is said to be convex if, for every $q \in P$, the tangent hyperplane $T_{q} P$ of $P$ at $q$ does not separate $P$ into two parts, see [13, Vol. II, p. 40], and [27, Vol III, p. 93]. Convexity extends to hypersurfaces in $\mathbb{K}_{b}^{n}, b \neq 0$, if in the above definition we replace 'the tangent hyperplane' by the totally geodesic hypersurface $\exp _{q}\left(T_{q} P\right)$ in $\mathbb{K}_{b}^{n}$, see [27, Vol IV, pp.121-123].

A classical generalization of Hadamard's Theorem states: If $P$ is a convex hypersurface in $\mathbb{K}_{b}^{n}$, then the Weingarten map of $P, L_{\xi}$, is semi-negative definite at all points $q \in P$, see [27, vol IV, pp.121-123 and vol III, pp. 91-95] and [2. Here $\xi$ denotes the outward pointing unit normal of the hypersurface. Therefore all the principal curvatures of $P$ will be non-positive according to the orientation chosen for $P$. Given $x \in P$, the outward pointing unit normal $\xi(x)$, and the mean curvature normal vector $H_{P}(x)$ to $P$ at $x$ we then obtain radial mean 0 -convexity of $P$ from any given point $p \in P$ :

$$
\begin{aligned}
\mathcal{C}(x) & =-\left\langle H_{P}(x), \nabla^{\mathbb{K}_{b}^{n}} r(x)\right\rangle \\
& =\frac{1}{n-1} \sum_{i=1}^{n-1}\left|k_{i}(x)\right|\left\langle\xi(x), \nabla^{\mathbb{K}_{b}^{n}} r(x)\right\rangle \\
& \geq 0,
\end{aligned}
$$

where $k_{i}(x)$ are the principal curvatures of $P$ at $x$ and we are using Proposition 2.3 in [24]. 
5.2. Lower Tangency Bounds. The final notion needed to describe our comparison setting is the idea of radial tangency. If we denote by $\nabla^{N} r$ and $\nabla^{P} r$ the gradients of $r$ in $N$ and $P$ respectively, then let us first remark that $\nabla^{P} r(q)$ is just the tangential component in $P$ of $\nabla^{N} r(q)$, for all $q \in P$. Hence we have the following basic relation:

$$
\nabla^{N} r=\nabla^{P} r+\left(\nabla^{P} r\right)^{\perp}
$$

where $\left(\nabla^{P} r\right)^{\perp}(q)$ is perpendicular to $T_{q} P$ for all $q \in P$.

Considering the extrinsic disc $D_{r(x)} \subset P$ and the outward pointing unit normal vector $\nu(x)$ to the extrinsic sphere $\partial D_{r(x)}$, then

$$
\nabla^{P} r(x)=\left\langle\nabla^{N} r(x), \nu(x)\right\rangle \nu(x),
$$

so that $\left\|\nabla^{P} r(x)\right\|$ measures the local tangency to $P$ at $x$ of the geodesics issuing from $p$. Full tangency means $\left\|\nabla^{P} r(x)\right\|=\left\langle\nabla^{N} r(x), \nu(x)\right\rangle=1$, i.e. $\nabla^{N} r(x)=\nu(x)$ and minimal tangency means orthogonality, i.e. $\left\|\nabla^{P} r(x)\right\|=\left\langle\nabla^{N} r(x), \nu(x)\right\rangle=0$.

In order to control this tangency of geodesics to the submanifold $P$ we introduce the following

Definition 5.3. We say that the submanifold $P$ satisfies a radial tangency condition from $p \in P$ when we have a smooth positive function $g: P \mapsto \mathbb{R}_{+}$, such that

$$
\mathcal{T}(x)=\left\|\nabla^{P} r(x)\right\| \geq g(r(x))>0 \quad \text { for all } \quad x \in P .
$$

5.3. Auxiliary Model Spaces. The concept of a model space is of instrumental importance for the precise statements of our comparison results. We therefore consider the definition and some first well-known properties in some detail:

Definition 5.4 (See [7], [6]). A $w$-model space $M_{w}^{m}$ is a smooth warped product with base $B^{1}=[0, R[\subset \mathbb{R}$ (where $0<R \leq \infty$ ), fiber $F^{m-1}=S_{1}^{m-1}$ (i.e. the unit $(m-1)$-sphere with standard metric), and warping function $w:\left[0, R\left[\rightarrow \mathbb{R}_{+} \cup\{0\}\right.\right.$ with $w(0)=0$, $w^{\prime}(0)=1$, and $w(r)>0$ for all $r>0$. The point $p_{w}=\pi^{-1}(0)$, where $\pi$ denotes the projection onto $B^{1}$, is called the center point of the model space. If $R=\infty$, then $p_{w}$ is a pole of $M_{w}^{m}$.

Remark 5.5. The simply connected space forms $\mathbb{K}_{b}^{m}$ of constant curvature $b$ can be constructed as $w$-models with any given point as center point using the warping functions

$$
w(r)=Q_{b}(r)=\left\{\begin{array}{ll}
\frac{1}{\sqrt{b}} \sin (\sqrt{b} r) & \text { if } b>0 \\
r & \text { if } b=0 \\
\frac{1}{\sqrt{-b}} \sinh (\sqrt{-b} r) & \text { if } b<0
\end{array} .\right.
$$

Note that for $b>0$ the function $Q_{b}(r)$ admits a smooth extension to $r=\pi / \sqrt{b}$. For $b \leq 0$ any center point is a pole. 
Proposition 5.6 (See 23 p. 206). Let $M_{w}^{m}$ be a $w$-model with warping function $w(r)$ and center $p_{w}$. The distance sphere of radius $r$ and center $p_{w}$ in $M_{w}^{m}$, denoted as $S_{r}^{w}$, is the fiber $\pi^{-1}(r)$. This distance sphere has the following constant mean curvature vector in $M_{w}^{m}$

$$
H_{\pi^{-1}(r)}=-\eta_{w}(r) \nabla^{M} \pi=-\eta_{w}(r) \nabla^{M} r,
$$

where the mean curvature function $\eta_{w}(r)$ is defined by

$$
\eta_{w}(r)=\frac{w^{\prime}(r)}{w(r)}=\frac{d}{d r} \ln (w(r)) .
$$

In particular we have for the constant curvature space forms $\mathbb{K}_{b}^{m}$ :

$$
\eta_{Q_{b}}(r)=\left\{\begin{array}{ll}
\sqrt{b} \cot (\sqrt{b} r) & \text { if } b>0 \\
1 / r & \text { if } b=0 \\
\sqrt{-b} \operatorname{coth}(\sqrt{-b} r) & \text { if } b<0
\end{array} .\right.
$$

Definition 5.7. Let $p$ be a point in a Riemannian manifold $M$ and let $x \in M-\{p\}$. The sectional curvature $K_{M}\left(\sigma_{x}\right)$ of the two-plane $\sigma_{x} \in T_{x} M$ is then called a $p$-radial sectional curvature of $M$ at $x$ if $\sigma_{x}$ contains the tangent vector to a minimal geodesic from $p$ to $x$. We denote these curvatures by $K_{p, M}\left(\sigma_{x}\right)$.

Proposition 5.8 (See [6] and [7]). Let $M_{w}^{m}$ be a $w$-model with center point $p_{w}$. Then the $p_{w}$-radial sectional curvatures of $M_{w}^{m}$ at every $x \in$ $\pi^{-1}(r)$ (for $r>0$ ) are all identical and determined by the radial function $K_{w}(r)$ defined as follows:

$$
K_{p_{w}, \tilde{N}_{w}}\left(\sigma_{x}\right)=K_{w}(r)=-\frac{w^{\prime \prime}(r)}{w(r)} .
$$

For any given warping function $w(r)$ we introduce the isoperimetric quotient function $q_{w}(r)$ for the corresponding $w$-model space $M_{w}^{m}$ as follows:

$$
q_{w}(r)=\frac{\operatorname{Vol}\left(B_{r}^{w}\right)}{\operatorname{Vol}\left(S_{r}^{w}\right)}=\frac{\int_{0}^{r} w^{m-1}(t) d t}{w^{m-1}(r)},
$$

where $B_{r}^{w}$ denotes the polar centered geodesic $r$-ball of radius $r$ in $M_{w}^{m}$ with boundary sphere $S_{r}^{w}$.

When discussing isoperimetric comparison inequalities the warping function $w$ usually appears raised to the power $m-1$. Accordingly we will use the notion $a_{w}(r)$ to denote this power of $w$ ( $V_{0}$ denotes the volume of the unit sphere in $\left.\mathbb{R}^{m}\right)$ :

$$
\begin{aligned}
& a_{w}(r)=\operatorname{Vol}\left(S_{r}^{w}\right) / V_{0}=w^{m-1}(r), \quad \text { so that } \\
& q_{w}(r)=\frac{\int_{0}^{r} a_{w}(t) d t}{a_{w}(r)} .
\end{aligned}
$$


5.4. Lower Curvature Bounds. The 2.nd order analysis of the restricted distance function $r_{\left.\right|_{P}}$ is firstly and foremost governed by the following Hessian comparison theorem for manifolds with a pole:

Theorem 5.9 (See [6], Theorem A). Let $N^{n}$ be a manifold with a pole $p$, let $M_{w}^{m}$ denote a $w$-model with center $p_{w}$. Suppose that every $p$ radial sectional curvature at $x \in N-\{p\}$ is bounded from above by the $p_{w}$-radial sectional curvatures in $M_{w}^{m}$ as follows:

$$
\mathcal{K}(\sigma(x))=K_{p, N}\left(\sigma_{x}\right) \geq-\frac{w^{\prime \prime}(r)}{w(r)}
$$

for every radial two-plane $\sigma_{x} \in T_{x} N$ at distance $r=r(x)=\operatorname{dist}_{N}(p, x)$ from $p$ in $N$. Then the Hessian of the distance function in $N$ satisfies

$$
\begin{aligned}
\operatorname{Hess}^{N}(r(x))(X, X) & \leq \operatorname{Hess}^{M_{w}^{m}}(r(y))(Y, Y) \\
& =\eta_{w}(r)\left(1-\left\langle\nabla^{M} r(y), Y\right\rangle_{M}^{2}\right) \\
& =\eta_{w}(r)\left(1-\left\langle\nabla^{N} r(x), X\right\rangle_{N}^{2}\right)
\end{aligned}
$$

for every unit vector $X$ in $T_{x} N$ and for every unit vector $Y$ in $T_{y} M$ with $r(y)=r(x)=r$ and $\left\langle\nabla^{M} r(y), Y\right\rangle_{M}=\left\langle\nabla^{N} r(x), X\right\rangle_{N}$.

If we let $\mu: N \mapsto \mathbb{R}$ denote a smooth function on $N$, then the restriction $\tilde{\mu}=\mu_{\left.\right|_{P}}$ is a smooth function on $P$ and the respective Hessian operators, $\operatorname{Hess}^{N}(\mu)$ and $\operatorname{Hess}^{P}(\tilde{\mu})$, are related as follows:

Proposition $5.10([10])$.

$$
\begin{aligned}
\operatorname{Hess}^{P}(\tilde{\mu})(X, Y)= & \operatorname{Hess}^{N}(\mu)(X, Y) \\
& +\left\langle\nabla^{N}(\mu), \alpha_{x}(X, Y)\right\rangle
\end{aligned}
$$

for all tangent vectors $X, Y \in T_{x} P^{m} \subset T_{x} N^{n}$, where $\alpha_{x}$ is the second fundamental form of $P$ at $x$ in $N$.

If we modify $\mu$ by a smooth function $f: \mathbb{R} \rightarrow \mathbb{R}$ we then get

Corollary 5.11 ([10]).

$$
\begin{aligned}
\operatorname{Hess}^{P}(f \circ \tilde{\mu})(X, X)= & f^{\prime \prime}(\mu)\left\langle\nabla^{N}(\mu), X\right\rangle^{2} \\
& +f^{\prime}(\mu) \operatorname{Hess}^{N}(\mu)(X, X) \\
& +\left\langle\nabla^{N}(\mu), \alpha_{x}(X, X)\right\rangle .
\end{aligned}
$$

for all $X \in T_{x} P^{m}$.

5.5. The Laplacian Comparison Space. Combining these results and tracing the resulting Hessian comparison statement in an orthonormal basis of $T_{x} P^{m}$, we obtain the following Laplacian inequality:

Corollary 5.12. Suppose again that the assumptions of Theorem 5.9 are satisfied. Then we have for every smooth function $f(r)$ with $f^{\prime}(r) \leq$ 
0 for all $r$, (respectively $f^{\prime}(r) \geq 0$ for all $\left.r\right)$ :

$$
\begin{aligned}
\Delta^{P}(f \circ r) \geq(\leq) & \left(f^{\prime \prime}(r)-f^{\prime}(r) \eta_{w}(r)\right)\left\|\nabla^{P} r\right\|^{2} \\
& +m f^{\prime}(r)\left(\eta_{w}(r)+\left\langle\nabla^{N} r, H_{P}\right\rangle\right),
\end{aligned}
$$

where $H_{P}$ denotes the mean curvature vector of $P$ in $N$.

5.6. The Isoperimetric Comparison Space. Given the tangency and convexity bounding functions $g(r), h(r)$ and the ambient curvature controller function $w(r)$ we construct a new model space $C_{w, g, h}^{m}$, which eventually will serve as the precise comparison space for the isoperimetric quotients of extrinsic balls in $P$.

Proposition 5.13. For the given smooth functions $w(r), g(r)$, and $h(r)$ defined above on the closed interval $[0, R]$, we consider the auxiliary function $\Lambda(r)$ for $r \in] 0, R]$, which is independent of $R$ and defined via the following equation. (We without lack of generality that $R>1$ which, if needed, can be obtained by scaling the metric of $N$ and thence $P$ by a constant.)

$$
\Lambda(r) w(r) g(r)=T \exp \left(-\int_{r}^{1} \frac{m}{g^{2}(t)}\left(\eta_{w}(t)-h(t)\right) d t\right),
$$

where $T$ is a positive constant - to be fixed shortly. Then there exists a unique smooth extension of $\Lambda(r)$ to the closed interval $[0, R]$ with $\Lambda(0)=0$. Moreover, the constant $T$ can be chosen so that

$$
\left.\frac{d}{d r}\right|_{r=0}\left(\Lambda^{\frac{1}{m-1}}(r)\right)=1
$$

Proof. Concerning the definition of $\Lambda$, the only problem is with the smooth extension to $r=0$. We first observe that the integrand of the right hand side of (5.18) can be expressed as follows - upon observing that $w(0)=0, w^{\prime}(0)=1$, and $g(0)=1$ :

$$
\frac{m}{g^{2}(t)}\left(\frac{w^{\prime}(t)}{w(t)}-h(t)\right)=\frac{m}{t}+\zeta(t)
$$

for a smooth, hence also bounded, function $\zeta(t)$ defined on the closed interval $[0, R]$.

Indeed, since $w(t)$ is assumed smooth in $[0, R]$ it means in particular, that the derivatives $w^{(n)}(0)=\lim _{t \rightarrow 0} w^{(n)}(t)$ are well defined for every 'derivation' number $n$. A repeated application of L'Hospital's rule together with the facts that $w(0)=0, w^{\prime}(0)=1$ then shows, that the function $\alpha(t)=w(t) / t$ is also a well defined bounded smooth positive function on the closed interval $[0, R]$. In particular $\alpha(0)=$ $\lim _{t \rightarrow 0}(w(t) / t)=1$. Then we have

$$
\beta(t)=\eta_{w}(t)-\frac{1}{t}=\frac{d}{d t}\left(\log \frac{w(t)}{t}\right)=\frac{\alpha^{\prime}(t)}{\alpha(t)}
$$


is a smooth bounded function on $[0, R]$. Yet another repeated application of L'Hospital's rule and the fact that $g(0)=1$, shows that the function

$$
\gamma(t)=\frac{g^{2}(t)}{t}-\frac{1}{t}
$$

is also a smooth bounded function on $[0, R]$.

The integrand of the right hand side of (5.18) can then be expressed as follows:

$$
\begin{aligned}
\frac{m}{g^{2}(t)}\left(\eta_{w}(t)-h(t)\right) & =\frac{m}{g^{2}(t)}\left(\frac{1}{t}+\beta(t)-h(t)\right) \\
& =\frac{m}{g^{2}(t)}\left(\frac{g^{2}(t)}{t}-\gamma(t)+\beta(t)-h(t)\right) \\
& =\frac{m}{t}+\zeta(t),
\end{aligned}
$$

where $\zeta(t)$ is a smooth bounded function on $[0, R]$ as claimed in $(5.20)$.

Equation (5.18) therefore reads for all $r \in[0, R]$ :

$$
\Lambda(r) w(r) g(r)=T r^{m} \exp \left(-\int_{r}^{1} \zeta(t) d t\right) .
$$

Hence applying once more the fact that $w(r)=r \alpha(r)$ we get:

$$
\Lambda(r)=T r^{m-1} G(r),
$$

where $G(r)$ is a smooth, bounded, positive function. Since $G(r)$ is smooth and positive the $(m-1)$ 'st root of that function is also smooth and positive throughout $[0, R]$. In other words:

$$
\Lambda^{\frac{1}{m-1}}(r)=r(T G(r))^{\frac{1}{m-1}}
$$

is a smooth, positive, bounded function on $[0, R]$ with $\Lambda^{\frac{1}{m-1}}(0)=0$, and the choice $T=1 / G(0)$ finally gives

$$
\left.\frac{d}{d r}\right|_{r=0}\left(\Lambda^{\frac{1}{m-1}}(r)\right)=1
$$

as needed.

The following observation is a direct consequence of the construction of $\Lambda(r)$ :

Lemma 5.14. The function $\Lambda(r)$ satisfies the following differential equation:

$$
\begin{aligned}
\frac{d}{d r} \Lambda(r) w(r) g(r) & =\Lambda(r) w(r) g(r)\left(\frac{m}{g^{2}(r)}\left(\eta_{w}(r)-h(r)\right)\right) \\
& =m \frac{\Lambda(r)}{g(r)}\left(w^{\prime}(r)-h(r) w(r)\right)
\end{aligned}
$$


Remark 5.15. In passing we also note that the function $\Lambda(r)$ defined as above is the essential ingredient in the solution to a Dirichlet-Poisson problem (7.4) to be considered below and is in this sense of instrumental importance for obtaining our main isoperimetric inequality in Theorem 7.1.

A 'stretching' function $s$ is defined as follows

$$
s(r)=\int_{0}^{r} \frac{1}{g(t)} d t
$$

It has a well-defined inverse $r(s)$ for $s \in[0, s(R)]$ with derivative $r^{\prime}(s)=g(r(s))$. In particular $r^{\prime}(0)=g(0)=1$.

With these concepts and key features of the auxiliary function $\Lambda(r)$ we are now able to define the carrier of the isoperimetric quotients to be compared with the isoperimetric quotients of the extrinsic balls $D_{R}$ :

Definition 5.16. The isoperimetric comparison space $C_{w, g, h}^{m}$ is the $W$-model space with base interval $B=[0, s(R)]$ and warping function $W(s)$ defined by

$$
W(s)=\Lambda^{\frac{1}{m-1}}(r(s))
$$

We observe firstly, that in spite of its relatively complicated construction, $C_{w, g, h}^{m}$ is indeed a model space with a well defined pole $p_{W}$ at $s=0: W(s) \geq 0$ for all $s$ and $W(s)$ is only 0 at $s=0$, where also, because of the explicit construction in Proposition 5.13 and equation (5.19): $W^{\prime}(0)=1$.

Secondly it should not be forgotten, that the spaces $C_{w, g, h}^{m}$ are specially tailor made to facilitate the proofs of the isoperimetric inequalities, that we are about to develop in section 7 as well as the explicit capacity comparison result in section 11 .

In order for this to work out we need one further particular property to be satisfied by these comparison spaces:

Of course any given $C_{w, g, h}^{m}$ inherits all its properties from the bounding functions $w, g$, and $h$ from which it is molded in the first place. Concerning the associated volume growth properties we note the following expressions for the isoperimetric quotient function:

Proposition 5.17. Let $B_{s}^{W}\left(p_{W}\right)$ denote the metric ball of radius $s$ centered at $p_{W}$ in $C_{w, g, h}^{m}$. Then the corresponding isoperimetric quotient 
function is

$$
\begin{aligned}
q_{W}(s) & =\frac{\operatorname{Vol}\left(B_{s}^{W}\left(p_{W}\right)\right)}{\operatorname{Vol}\left(\partial B_{s}^{W}\left(p_{W}\right)\right)} \\
& =\frac{\int_{0}^{s} W^{m-1}(t) d t}{W^{m-1}(s)} \\
& =\frac{\int_{0}^{r(s)} \frac{\Lambda(u)}{g(u)} d u}{\Lambda(r(s))} .
\end{aligned}
$$

\subsection{A Balance Condition.}

Definition 5.18. The model space $M_{W}^{m}=C_{w, g, h}^{m}$ is $w$-balanced from below (with respect to the intermediary model space $M_{w}^{m}$ ) if the following holds for all $r \in[0, R]$, resp. all $s \in[0, s(R)]$ :

$$
m q_{W}(s)\left(\eta_{w}(r(s))-h(r(s))\right) \geq g(r(s))
$$

Remark 5.19. In particular the $w$-balance condition implies that

$$
\eta_{w}(r)-h(r)>0
$$

The above definition of a $w$-balance condition from below is an extension of the balance condition from below as applied in [21]. The condition there reads as follows and is obtained from (5.32) precisely when $g(r)=1$ and $h(r)=0$ for all $r \in[0, R]$ so that $r(s)=s$, $W(s)=w(r):$

$$
m q_{w}(r) \eta_{w}(r) \geq 1
$$

Lemma 5.20. The balance condition (5.32) is equivalent to the following inequality:

$$
m\left(\int_{0}^{r} \frac{\Lambda(t)}{g(t)} d t\right)\left(w^{\prime}(r)-h(r) w(r)\right) \geq \Lambda(r) w(r) g(r)
$$

Moreover, the balance condition (5.32), hence (5.35), is implied by the following (thence stronger) inequality, which does not involve the tangency bounding function $g(r)$ :

$$
w^{\prime \prime}(r)-w^{\prime}(r) h(r)-w(r) h^{\prime}(r) \geq 0 .
$$


Proof. A direct differentiation as in (5.31) but with respect to $r$ amounts to:

$$
\begin{aligned}
& \frac{d}{d r}\left(\frac{q_{W}(s(r))}{g(r) w(r)}\right)=\frac{d}{d r}\left(\frac{\int_{0}^{r} \frac{\Lambda(u)}{g(u)} d u}{\Lambda(r) g(r) w(r)}\right) \\
& =\frac{\Lambda(r)}{g^{2}(r) \Lambda(r) w(r)}-\frac{\frac{d}{d r}(\Lambda(r) g(r) w(r)) \int_{0}^{r} \frac{\Lambda(u)}{g(u)} d u}{\Lambda^{2}(r) g^{2}(r) w^{2}(r)} \\
& =\frac{1}{\Lambda(r) g(r) w(r)}\left(\frac{\Lambda(r)}{g(r)}-\left(\frac{m}{g^{2}(r)}\left(\eta_{w}-h(r)\right)\right) \int_{0}^{r} \frac{\Lambda(u)}{g(u)} d u\right) \\
& =\frac{1}{\Lambda(r) g^{2}(r) w^{2}(r)}\left(\Lambda(r) w(r) g(r)-m\left(\int_{0}^{r} \frac{\Lambda(t)}{g(t)} d t\right)\left(w^{\prime}(r)-h(r) w(r)\right)\right)
\end{aligned}
$$

which shows that 5.32 and 5.35 are equivalent.

Now let $G(r)$ denote the left hand side of the balance condition inequality (5.35), and let $F(r)$ denote the right hand side:

$G(r)=m\left(\int_{0}^{r} \frac{\Lambda(t)}{g(t)} d t\right)\left(w^{\prime}(r)-h(r) w(r)\right) \geq \Lambda(r) w(r) g(r)=F(r)$

Then $G(0)=F(0)=0$ and, moreover

$$
\begin{aligned}
G^{\prime}(r) & =m \frac{\Lambda(r)}{g(r)}\left(w^{\prime}(r)-h(r) w(r)\right) \\
& +m\left(\int_{0}^{r} \frac{\Lambda(r)}{g(r)} d t\right)\left(w^{\prime \prime}(r)-w^{\prime}(r) h(r)-w(r) h^{\prime}(r)\right) \\
& \geq m \frac{\Lambda(r)}{g(r)}\left(w^{\prime}(r)-h(r) w(r)\right) \\
& =F^{\prime}(r),
\end{aligned}
$$

where we have used Lemma 5.14 (for $F^{\prime}(r)$ ) and the balance condition (5.36). It follows that $G(r) \geq F(r)$ for all $r \in[0, R]$, and this proves the Lemma.

\subsection{Balance on the Edge.}

Lemma 5.21. Equality in the balance condition (5.32) is equivalent to equality in the stronger condition (5.36) and equivalent to each one of the following identities:

$$
\begin{gathered}
\eta_{w}(r)-h(r)=\frac{1}{w(r)} \\
\Lambda(r) w(r) g(r)=m \int_{0}^{r} \frac{\Lambda(t)}{g(t)} d t . \\
q_{W}(s)=\frac{1}{m} w(r(s)) g(r(s)) .
\end{gathered}
$$


Proof. From equality in (5.36) we get

$$
w^{\prime}(r)=w(r) h(r)+c
$$

for some constant $c$ which must then be $c=1$ since $w^{\prime}(0)=1$. Then $w^{\prime}(r)-w(r) h(r)=0$ gives identity in equation (5.37) as well and vice versa.

Remark 5.22. Special cases of equality in the balance condition are obtained by $h(r)=C$ and $w(r)=r$. This corresponds to the situation considered in section 4 - where we analyzed radially mean 0 -convex surfaces in Euclidean 3-space.

\section{The Isoperimetric Comparison Constellation}

The intermediate observations considered above together with the previously introduced bounds on radial curvature and tangency now constitute the notion of a comparison constellation (for the isoperimetric inequality) as follows.

Definition 6.1. Let $N^{n}$ denote a Riemannian manifold with a pole $p$ and distance function $r=r(x)=\operatorname{dist}_{N}(p, x)$. Let $P^{m}$ denote an unbounded complete and closed submanifold in $N^{n}$. Suppose $p \in P^{m}$, and suppose that the following conditions are satisfied for all $x \in P^{m}$ with $r(x) \in[0, R]$ :

(a) The $p$-radial sectional curvatures of $N$ are bounded from below by the $p_{w}$-radial sectional curvatures of the $w$-model space $M_{w}^{m}$ :

$$
\mathcal{K}\left(\sigma_{x}\right) \geq-\frac{w^{\prime \prime}(r(x))}{w(r(x))}
$$

(b) The $p$-radial mean curvature of $P$ is bounded from below by a positive smooth radial function $h(r)$ :

$$
\mathcal{C}(x) \geq h(r(x)) \text {. }
$$

(c) The $p$-radial tangency of $P$ is bounded from below by a smooth radial function $g(r)$ :

$$
\mathcal{T}(x) \geq g(r(x))>0 .
$$

Let $C_{w, g, h}^{m}$ denote the $W$-model with the specific warping function $W$ : $\pi\left(C_{w, g, h}^{m}\right) \rightarrow \mathbb{R}_{+}$which is constructed above in Definition 5.16 via $w$, $g$, and $h$. Then the triple $\left\{N^{n}, P^{m}, C_{w, g, h}^{m}\right\}$ is called an isoperimetric comparison constellation on the interval $[0, R]$.

\section{MAIN ISOPERIMETRIC RESUltS}

In this section we find upper bounds for the isoperimetric quotient defined as the volume of the extrinsic sphere divided by the volume of the extrinsic ball, in the setting given by the comparison constellations defined in Definition 6.1: 
Theorem 7.1. We consider an isoperimetric comparison constellation $\left\{N^{n}, P^{m}, C_{w, g, h}^{m}\right\}$ on the interval $[0, R]$. Suppose further that the comparison space $C_{w, g, h}^{m}$ is $w$-balanced from below in the sense of Definition (5.18). Then

$$
\frac{\operatorname{Vol}\left(\partial D_{R}\right)}{\operatorname{Vol}\left(D_{R}\right)} \leq \frac{\operatorname{Vol}\left(\partial B_{s(R)}^{W}\right)}{\operatorname{Vol}\left(B_{s(R)}^{W}\right)} \leq \frac{m}{g(R)}\left(\eta_{w}(R)-h(R)\right)
$$

If the comparison space $C_{w, g, h}^{m}$ satisfies the balance condition with equality in (5.32) (or equivalently in (5.36)) for all $r \in[0, R]$, then

$$
\frac{\operatorname{Vol}\left(\partial D_{R}\right)}{\operatorname{Vol}\left(D_{R}\right)} \leq \frac{\operatorname{Vol}\left(\partial B_{s(R)}^{W}\right)}{\operatorname{Vol}\left(B_{s(R)}^{W}\right)}=\frac{m}{w(R) g(R)} .
$$

Remark 7.2. We first note here, that Theorem A follows readily from this result, since the $w$-balance condition holds (with equality) in the simple situation of Theorem A.

Proof. We define a second order differential operator L on functions $f$ of one real variable as follows:

$$
\mathrm{L} f(r)=f^{\prime \prime}(r) g^{2}(r)+f^{\prime}(r)\left(\left(m-g^{2}(r)\right) \eta_{w}(r)-m h(r)\right),
$$

and consider the smooth solution $\psi(r)$ to the following Dirichlet-Poisson problem:

$$
\begin{aligned}
\mathrm{L} \psi(r) & =-1 \text { on }[0, R] \\
\psi(R) & =0
\end{aligned}
$$

The ODE problem is equivalent to the following:

$$
\psi^{\prime \prime}(r)+\psi^{\prime}(r)\left(-\eta_{w}(r)+\frac{m}{g^{2}(r)}\left(\eta_{w}(r)-h(r)\right)\right)=-\frac{1}{g^{2}(r)} .
$$

The solution is constructed via the auxiliary function $\Lambda(r)$ from (5.18) as follows:

$$
\begin{aligned}
\psi^{\prime}(r) & =\Gamma(r) \\
& =\exp (-\mathcal{P}(r)) \int_{0}^{r} \exp (\mathcal{P}(t))\left(-\frac{1}{g^{2}(t)}\right) d t,
\end{aligned}
$$

where the auxiliary function $\mathcal{P}$ is defined as follows, assuming again without lack of generality, that $R>1$, so that the domain of definition of all involved functions contains $[0,1]$ :

$$
\begin{aligned}
\mathcal{P}(r) & =\int_{r}^{1}\left(\eta_{w}(t)-\frac{m}{g^{2}(t)}\left(\eta_{w}(t)-h(t)\right)\right) d t \\
& =\log \left(\frac{w(1)}{w(r)}\right)-\int_{r}^{1} \frac{m}{g^{2}(t)}\left(\eta_{w}(t)-h(t)\right) d t .
\end{aligned}
$$


Hence

$$
\begin{aligned}
& \exp (\mathcal{P}(t))\left(\frac{1}{g^{2}(t)}\right)= \\
& \frac{w(1)}{w(t) g^{2}(t)} \exp \left(-\int_{t}^{1} \frac{m}{g^{2}(u)}\left(\eta_{w}(u)-h(u)\right) d u\right) \\
& =\frac{w(1)}{w(t) g^{2}(t) T} \Lambda(t) w(t) g(t)=\left(\frac{w(1)}{T}\right)\left(\frac{\Lambda(t)}{g(t)}\right) .
\end{aligned}
$$

From this latter expression we then have as well

$$
\exp (-\mathcal{P}(r))=\left(\frac{T}{w(1)}\right)\left(\frac{1}{g(r) \Lambda(r)}\right),
$$

so that

$$
\begin{aligned}
\psi^{\prime}(r) & =\frac{-1}{g(r) \Lambda(r)} \int_{0}^{r} \frac{\Lambda(t)}{g(t)} d t \\
& =-\frac{\operatorname{Vol}\left(B_{s(r)}^{W}\right)}{g(r) \operatorname{Vol}\left(\partial B_{s(r)}^{W}\right)} \\
& =-\frac{q_{W}(s(r))}{g(r)} .
\end{aligned}
$$

At this instance we must observe, that it follows clearly from this latter expression for $\psi^{\prime}(r)$ that the above formal, yet standard, method of solving (7.4) indeed does produce a smooth solution on $[0, R]$ with $\psi^{\prime}(0)=0$. Then we have:

$$
\begin{aligned}
\psi(r) & =\int_{r}^{R} \frac{1}{g(u) \Lambda(u)}\left(\int_{0}^{u} \frac{\Lambda(t)}{g(t)} d t\right) d u \\
& =\int_{r}^{R} \frac{q_{W}(s(u))}{g(u)} d u \\
& =\int_{s(r)}^{s(R)} q_{W}(t) d t .
\end{aligned}
$$

We now show that - because of the balance condition (5.36) - the function $\psi(r)$ enjoys the following inequality:

\section{Lemma 7.3.}

$$
\psi^{\prime \prime}(r)-\psi^{\prime}(r) \eta_{w}(r) \geq 0
$$

Proof of Lemma. We must show that

$$
\Gamma^{\prime}(r)-\Gamma(r) \eta_{w}(r) \geq 0
$$


Since

$$
\begin{aligned}
\Gamma^{\prime}(r) & =\psi^{\prime \prime}(r) \\
& =-\frac{1}{g^{2}(r)}\left(1+\psi^{\prime}(r)\left(\left(m-g^{2}(r)\right) \eta_{w}(r)-m h(r)\right)\right) \\
& =-\frac{1}{g^{2}(r)}\left(1+\Gamma(r)\left(\left(m-g^{2}(r)\right) \eta_{w}(r)-m h(r)\right)\right)
\end{aligned}
$$

we have

$$
\Gamma^{\prime}(r)-\Gamma(r) \eta_{w}(r)=-\frac{1}{g^{2}(r)}\left(1+m \Gamma(r)\left(\eta_{w}(r)-h(r)\right)\right)
$$

Therefore equation (7.13) is equivalent to each one of the following inequalities, observing that $\Gamma(r)<0$ for all $r \in] 0, R]$ :

$$
\begin{gathered}
\frac{m}{g(r) \Lambda(r)}\left(\int_{0}^{r} \frac{\Lambda(t)}{g(t)} d t\right)\left(\frac{w^{\prime}(r)}{w(r)}-h(r)\right) \geq 1, \\
m\left(\int_{0}^{r} \frac{\Lambda(t)}{g(t)} d t\right)\left(w^{\prime}(r)-h(r) w(r)\right) \geq \Lambda(r) w(r) g(r),
\end{gathered}
$$

where the latter inequality is equivalent to 5.32 via Lemma 5.20

With Lemma 7.3 in hand we continue the proof of Theorem 7.1. Applying the Laplace inequality (5.17) for the function $\psi(r)$ transplanted into $P^{m}$ in $N^{n}$ now gives the following comparison

$$
\begin{aligned}
\Delta^{P} \psi(r(x)) \geq & \left(\psi^{\prime \prime}(r(x))-\psi^{\prime}(r(x)) \eta_{w}(r(x))\right) g^{2}(r(x)) \\
& \quad+m \psi^{\prime}(r(x))\left(\eta_{w}(r(x))-h(r(x))\right) \\
= & \operatorname{L~} \psi(r(x)) \\
= & -1 \\
= & \Delta^{P} E(x),
\end{aligned}
$$

where $E(x)$ is the mean exit time function for the extrinsic ball $D_{R}$, with $E_{\left.\right|_{\partial D_{R}}}=0$. The maximum principle then applies and gives:

$$
E(x) \geq \psi(r(x)), \text { for all } x \in D_{R} .
$$


Applying the divergence theorem, taking the unit normal to $\partial D_{R}$ as $\frac{\nabla^{P} r}{\left\|\nabla^{P} r\right\|}$, we get

$$
\begin{aligned}
\operatorname{Vol}\left(D_{R}\right) & =\int_{D_{R}}-\Delta^{P} u(x) d \mu \\
& \geq \int_{D_{R}}-\Delta^{P} \psi(r(x)) d \mu \\
& =-\int_{D_{R}} \operatorname{div}\left(\nabla^{P} \psi(r(x))\right) d \mu \\
& =-\int_{\partial D_{R}}<\nabla^{P} \psi(r(x)), \frac{\nabla^{P} r(x)}{\left\|\nabla^{P} r\right\|}>d \nu \\
& =-\Gamma(R) \int_{\partial D_{R}}\left\|\nabla^{P} r\right\| d \nu \\
& \geq-\Gamma(R) g(R) \operatorname{Vol}\left(\partial D_{R}\right),
\end{aligned}
$$

which shows the isoperimetric inequality (7.1).

We finally consider the case of equality in the balance condition, which implies in particular that (5.39) holds true. Using this we have immediately, as claimed:

$$
\frac{\operatorname{Vol}\left(\partial B_{s(R)}^{W}\right)}{\operatorname{Vol}\left(B_{s(R)}^{W}\right)}=\frac{\Lambda(R)}{\int_{0}^{R} \frac{\Lambda(t)}{g(t)} d t}=\frac{m}{g(R) w(R)} .
$$

\section{Consequences}

We first observe the following volume comparison in consequence of Theorem 7.1, inequality (7.1):

Corollary 8.1. Let $\left\{N^{n}, P^{m}, C_{w, g, h}^{m}\right\}$ be a comparison constellation on the interval $[0, R]$, as in Theorem [7.1. Then

$$
\operatorname{Vol}\left(D_{r}\right) \leq \operatorname{Vol}\left(B_{s(r)}^{W}\right) \quad \text { for every } r \in[0, R] .
$$

Proof. Let $\mathcal{G}(r)$ denote the following function

$$
\mathcal{G}(r)=\log \left(\frac{\operatorname{Vol}\left(D_{r}\right)}{\operatorname{Vol}\left(B_{s(r)}^{W}\right)}\right) .
$$

Since $W(s)$ is a warping function for an $m$-dimensional model space we have $\mathcal{G}(0)=\lim _{r \rightarrow 0} \mathcal{G}(r)=0$ and furthermore

$$
\mathcal{G}^{\prime}(r)=\left(\frac{\frac{\partial}{\partial r} \operatorname{Vol}\left(D_{r}\right)}{\operatorname{Vol}\left(D_{r}\right)}\right)-\left(\frac{\frac{\partial}{\partial s} \operatorname{Vol}\left(B_{s}^{W}\right)}{g(r) \operatorname{Vol}\left(B_{s(r)}^{W}\right)}\right) .
$$


From the co-area formula (see below) we have:

$$
g(r) \frac{\partial}{\partial r} \operatorname{Vol}\left(D_{r}\right) \leq \operatorname{Vol}\left(\partial D_{r}\right)
$$

so that the isoperimetric inequality 7.1 gives:

$$
\mathcal{G}^{\prime}(r) \leq \frac{1}{g(r)}\left(\frac{\operatorname{Vol}\left(\partial D_{r}\right)}{\operatorname{Vol}\left(D_{r}\right)}-\frac{\operatorname{Vol}\left(\partial B_{s(r)}^{W}\right)}{\operatorname{Vol}\left(B_{s(r)}^{W}\right)}\right) \leq 0
$$

In consequence we therefore have $\mathcal{G}(r) \leq \mathcal{G}(0)=0$, or equivalently:

$$
\operatorname{Vol}\left(D_{r}\right) \leq \operatorname{Vol}\left(B_{s(r)}^{W}\right) \quad \text { for every } r \in[0, R] .
$$

Proposition 8.2. Let $\left\{N^{n}, P^{m}, C_{w, g, h}^{m}\right\}$ be a comparison constellation on the interval $[0, R]$, as in Theorem 7.1 . Then we have:

$$
g(r) \frac{\partial}{\partial r} \operatorname{Vol}\left(D_{r}\right) \leq \operatorname{Vol}\left(\partial D_{r}\right) \quad \text { for all } \quad r \in[0, R] .
$$

Proof. Let $\Psi(x)=\psi(r(x))$ denote the radial mean exit time function, transplanted into $D_{R}$.

With the notation of [1] we then have

$$
\begin{aligned}
\Omega(t) & =\{x \in P \mid \psi(r(x))>t\}=D_{\psi^{-1}(t)} \\
V(t) & =\operatorname{Vol}\left(D_{\psi^{-1}(t)}\right) \\
\text { and } \Sigma(t) & \left.=\partial D_{\psi^{-1}(t)} \text { for all } t \in\right] 0, \psi(0)[.
\end{aligned}
$$

The co-area formula states that

$$
V^{\prime}(t)=-\int_{\partial D_{\psi^{-1}(t)}}\left\|\nabla^{P} \Psi(x)\right\|^{-1} d \sigma_{t} .
$$

On the other hand, we know that on $D_{R}$ we have for all $r$ :

$$
\left\|\nabla^{P} \Psi\right\|=-\Gamma(r)\left\|\nabla^{P} r\right\| \geq-g(r) \Gamma(r),
$$

so we then have:

$$
-\left\|\nabla^{P} \Psi\right\|^{-1} \geq \frac{1}{g(r) \Gamma(r)}
$$

Therefore

$$
\begin{aligned}
V^{\prime}(t) & \geq \int_{\partial D_{\psi^{-1}(t)}} \frac{1}{g(r) \Gamma(r)} d \sigma_{t} \\
& =\frac{1}{g(r) \Gamma(r)} \operatorname{Vol}\left(\partial D_{\psi^{-1}(t)}\right) .
\end{aligned}
$$

We define $F(r)=\operatorname{Vol}\left(D_{r}\right)$ and have

$$
V(t)=\operatorname{Vol}\left(D_{\psi^{-1}(t)}\right)=F \circ \psi^{-1}(t),
$$


then

$$
\begin{aligned}
V^{\prime}(t) & =F^{\prime}\left(\psi^{-1}(t)\right) \frac{d}{d t} \psi^{-1}(t) \\
& =\frac{\frac{d}{d r} \operatorname{Vol}\left(D_{r}\right)}{\frac{d}{d r} \psi(r)} \\
& =\frac{\frac{d}{d r} \operatorname{Vol}\left(D_{r}\right)}{\Gamma(r)}
\end{aligned}
$$

Since we also know from equation 8.11 that

$$
V^{\prime}(t) \geq \frac{\operatorname{Vol}\left(\partial D_{r}\right)}{g(r) \Gamma(r)}
$$

and since $\Gamma(r)<0$ on $] 0, R[$, we finally get, as claimed:

$$
g(r) \frac{d}{d r} \operatorname{Vol}\left(D_{r}\right) \leq \operatorname{Vol}\left(\partial D_{r}\right) \text { for all } r \in[0, R] .
$$

Corollary 8.3. Let $\left\{N^{n}, P^{m}, C_{w, g, h}^{m}\right\}$ be a comparison constellation on $[0, R]$, as in Theorem 7.1 . Then

$$
\int_{\partial D_{r}}\left\|\nabla^{P} r\right\| d \nu \leq g(r) W^{m-1}(s(r)) \text { for every } r \in[0, R] .
$$

Proof. From the proof of the isoperimetric inequality, Theorem 7.1, we extract the following inequality from equation (7.21)

$$
\int_{\partial D_{\rho}}\left\|\nabla^{P} r\right\| d \nu \leq \frac{\operatorname{Vol}\left(D_{\rho}\right)}{-\Gamma(\rho)}
$$

From the previous Corollary 8.1 we have

$$
\operatorname{Vol}\left(D_{\rho}\right) \leq \operatorname{Vol}\left(B_{s(\rho)}^{W}\right)
$$

so that

$$
\int_{\partial D_{\rho}}\left\|\nabla^{P} r\right\| d \nu \leq \frac{\operatorname{Vol}\left(B_{s(\rho)}^{W}\right)}{-\Gamma(\rho)}=g(\rho) \Lambda(\rho)
$$

When the submanifold $P^{m}$ is minimal and the ambient space $N^{n}$ is a Cartan-Hadamard manifold (with sectional curvatures bounded from above by 0 ), we get the following two-sided isoperimetric inequality:

Corollary 8.4. Let $P^{m}$ be a minimal submanifold in $N^{n}$. Let $p \in P^{m}$ be a point which is a pole of $N$. Suppose that the p-radial sectional curvatures of $N$ are bounded from above and from below as follows:

$$
-\frac{w_{2}^{\prime \prime}(r(x))}{w_{2}(r(x))} \leq \mathcal{K}\left(\sigma_{x}\right) \leq-\frac{w_{1}^{\prime \prime}(r(x))}{w_{1}(r(x))} \leq 0 .
$$


Then

$$
\frac{\operatorname{Vol}\left(\partial B_{R}^{w_{1}}\right)}{\operatorname{Vol}\left(B_{R}^{w_{1}}\right)} \leq \frac{\operatorname{Vol}\left(\partial D_{R}\right)}{\operatorname{Vol}\left(D_{R}\right)} \leq \frac{\operatorname{Vol}\left(\partial B_{s(R)}^{W_{2}}\right)}{\operatorname{Vol}\left(B_{s(R)}^{W_{2}}\right)} \leq \frac{m}{g(R)}\left(\eta_{w_{2}}(R)-h(R)\right),
$$

where $W_{2}(s)$ is the warping function of the comparison model space $C_{w_{2}, g, 0}^{m}$, see Theorem 7.1.

Proof. Since $P$ is minimal it is radially mean 0 -convex, so $h=0$. The upper isoperimetric bound follows then directly from Theorem 7.1. Indeed, $\left\{N^{n}, P^{m}, C_{w_{2}, g, 0}^{m}\right\}$ is a comparison constellation with the model comparison space $C_{w_{2}, q, 0}^{m}$ which is $w_{2}$-balanced from below. This latter claim follows because $w_{2}(r)$ satisfies the strong balance condition (5.36) in view of the assumptions $-w_{2}^{\prime \prime}(r(x)) / w_{2}(r(x)) \leq 0$ and $h=0$. The lower isoperimetric bound follows directly from Theorem B in [21]. Also for this to hold we need the corresponding balance condition from below, which is again satisfied because of the curvature assumption: $-w_{1}^{\prime \prime}(r(x)) / w_{1}(r(x)) \leq 0$, see [21, Observation 5.12].

\section{The Intrinsic Viewpoint}

In this section we consider the intrinsic versions of the isoperimetric and volume comparison inequalities (7.1) and (8.1) assuming that $P^{m}=N^{n}$. In this case, the extrinsic distance to the pole $p$ becomes the intrinsic distance in the ambient manifold, so, for all $r>0$ the extrinsic domains $D_{r}$ become the geodesic balls $B_{r}^{N}$ of $N^{n}$. We have for all $x \in P$ :

$$
\begin{aligned}
\nabla^{P} r(x) & =\nabla^{N} r(x) \\
H_{P}(x) & =0 .
\end{aligned}
$$

Thus $\left\|\nabla^{P} r\right\|=1$, so $g(r(x))=1$ and $\mathcal{C}(x)=h(r(x))=0$, the stretching function becomes the identity $s(r)=r, W(s(r))=w(r)$, and the isoperimetric comparison space $C_{w, g, h}^{m}$ is reduced to the auxiliary model space $M_{w}^{m}$. Applying Proposition 5.13 we then have

Corollary 9.1. Let $N^{n}$ denote a complete riemannian manifold with a pole $p$. Suppose that the p-radial sectional curvatures of $N^{n}$ are bounded from below by the $p_{w}$-radial sectional curvatures of a $w$-model space $M_{w}^{n}$ for all $r>0$. Then

$$
\frac{\operatorname{Vol}\left(\partial B_{R}^{N}\right)}{\operatorname{Vol}\left(B_{R}^{N}\right)} \leq \frac{\operatorname{Vol}\left(\partial B_{R}^{w}\right)}{\operatorname{Vol}\left(B_{R}^{w}\right)} \leq n \eta_{w}(R)
$$

Proof. The proof follows along the lines of theorem 7.1. Indeed, since $g(r)=1$ and $h(r)=0$, the second order differential operator $L$ agrees with the Laplacian on functions of one real variable defined on the model spaces $M_{w}^{n}$, namely,

$$
L f(r)=f^{\prime \prime}(r)+(n-1) \eta_{w}(r) f^{\prime}(r)
$$


Solving the corresponding problem $(7.4)$ on $[0, R]$ under these conditions and transplanting the solution $\psi(r)$ to the geodesic ball $B_{R}^{N}$, we can apply the Laplacian comparison analysis using formally $H_{P}=0$ because $P=N$. Then, we obtain the inequality

$$
\Delta^{N} \psi(r(x)) \geq-1=\Delta^{N} E(x)
$$

As a consequence of equality $\left\|\nabla^{P} r\right\|=1$, inequality $(7.12)$ is obsolete for the statement of inequality (7.19) to hold true. Hence, we do not need the balance condition as hypothesis, and thence the conclusion follows as in the proof of Theorem 7.1, applying the divergence theorem together with the inequality 9.4 .

The well-known volume comparison is a straightforward consequence, see [26], and recovered as follows:

Corollary 9.2. Let $N^{n}$ denote a complete Riemannian manifold with a pole $p$. Suppose that the p-radial sectional curvatures of $N^{n}$ are bounded from below by the $p_{w}$-radial sectional curvatures of a $w$-model space $M_{w}^{n}$ for all $r>0$. Then

$$
\operatorname{Vol}\left(B_{r}^{N}\right) \leq \operatorname{Vol}\left(B_{r}^{w}\right) \quad \text { for every } r \in[0, R] .
$$

Proof. We follow the lines of the proof of Corollary 8.1, but now letting $\mathcal{G}(r)$ denote the following function:

$$
\mathcal{G}(r)=\log \left(\frac{\operatorname{Vol}\left(D_{r}\right)}{\operatorname{Vol}\left(B_{r}^{w}\right)}\right) .
$$

Taking into account that $\left\|\nabla^{P} r\right\|=\left\|\nabla^{N} r\right\|=1=g$, the co-area formula gives the following known equality (the intrinsic version of Proposition 8.2 .

$$
\frac{\partial}{\partial r} \operatorname{Vol}\left(B_{r}^{N}\right)=\operatorname{Vol}\left(\partial B_{r}^{N}\right) \quad \text { for all } \quad r \in[0, R] .
$$

so the isoperimetric inequality 9.2 gives

$$
\mathcal{G}^{\prime}(r) \leq 0 \quad,
$$

and hence the volume comparison follows.

The intrinsic version of Corollary 8.3 gives likewise the following well-known inequality for the volume of extrinsic spheres, see again [26]:

Corollary 9.3. Let $N^{n}$ denote a complete Riemannian manifold with a pole $p$. Suppose that the p-radial sectional curvatures of $N^{n}$ are bounded from below by the $p_{w}$-radial sectional curvatures of a w-model space $M_{w}^{n}$ for all $r>0$. Then

$$
\operatorname{Vol}\left(\partial D_{r}\right) \leq w^{n-1}(r)=\operatorname{Vol}\left(\partial B_{r}^{w}\right) \text { for every } r \in[0, R] .
$$




\section{Mean Exit Time Bounds And a Non-explosion Criterion}

The solution $E$ to the Dirichlet-Poisson problem

$$
\begin{aligned}
\Delta E & =-1 \text { on } D_{R} \\
E_{\left.\right|_{\partial D_{R}}} & =0
\end{aligned}
$$

is, as already alluded to in the proof of Theorem 7.1, equation (7.19), the mean exit time for Brownian diffusion in $D_{R}$.

A. Gray and M. Pinsky proved in [4] a nice result, which even in the following truncated version shows in effect, that Brownian diffusion is fast in negative curvature and slow in positive curvature, even on the level of scalar curvature:

Theorem 10.1 (Gray and Pinsky). Let $B_{r}^{m}(p)$ denote an intrinsic geodesic ball of small radius $r$ and center $p$ in a Riemannian manifold $\left(M^{m}, g\right)$ which has scalar curvature $\tau(p)$ at the center point $p$. Then the mean exit time for Brownian particles starting at $p$ is

$$
E_{r}(p)=\frac{r^{2}}{2 m}+\frac{\tau(p) r^{4}}{12 m^{2}(m+2)}+r^{6} \varepsilon(r),
$$

where $\varepsilon(r) \rightarrow 0$ when $r \rightarrow 0$.

In view of this reference to the work by Gray and Pinsky [4 we mention here the following consequence of the proof of Theorem 7.1 . equations 7.20 and 7.11.

Theorem 10.2. Let $\left\{N^{n}, P^{m}, C_{w, g, h}^{m}\right\}$ denote an isoperimetric comparison constellation which is $w$-balanced from below on $[0, R]$. Then for all $x \in D_{R} \subset P^{m}$, we have:

$$
E(x) \geq E_{W}(s(r(x)))=\int_{s(r(x))}^{s(R)} q_{W}(t) d t,
$$

where $E_{W}(s)$ is the mean exit time function for Brownian motion in the disc of radius $R$ centered at the pole $p_{W}$ in the model space $C_{w, g, h}^{m}$. The function $q_{W}(s)$ is the isoperimetric quotient function and $s(r)$ is the stretching function of the $W$-model comparison space.

Moreover, as direct applications of the volume comparison in Corollary 8.1, using the obvious fact that the geodesic balls in $P, B_{R}^{P}$ are subsets of the extrinsic balls $D_{R}$ of the same radius, we get from the general non-explosion condition and parabolicity condition in [7, Theorem 9.1]:

Proposition 10.3. Let $\left\{N^{n}, P^{m}, C_{w, g, h}^{m}\right\}$ denote an isoperimetric comparison constellation which is $w$-balanced from below on $[0, \infty[$. Suppose that

$$
\int^{\infty} \frac{r(s) g(r(s))}{\log \left(\operatorname{Vol}\left(B_{s}^{W}\right)\right)} d s=\infty
$$


Then $P^{m}$ is stochastically complete, i.e. the Brownian motion in the submanifold is non-explosive.

From the same volume comparison result we get

Proposition 10.4. Let $\left\{N^{n}, P^{m}, C_{w, g, h}^{m}\right\}$ denote an isoperimetric comparison constellation which is $w$-balanced from below on $[0, \infty[$. Suppose that

$$
\int^{\infty} \frac{r(s) g(r(s))}{\operatorname{Vol}\left(B_{s}^{W}\right)} d s=\infty
$$

Then $P^{m}$ is parabolic.

We also have the following extrinsic version of Ichihara's [8, Theorem $2.1]$.

Theorem 10.5. Let $\left\{N^{n}, P^{m}, C_{w, g, h}^{m}\right\}$ denote an isoperimetric comparison constellation which is $w$-balanced from below on $[0, \infty[$. Suppose that

$$
\int^{\infty} \frac{1}{\operatorname{Vol}\left(\partial B_{s}^{W}\right)} d s=\infty
$$

Then $P^{m}$ is parabolic.

Remark 10.6. We shall prove this statement in the next section via a capacity comparison technique, which is very similar to the isoperimetric comparison developed in the previous sections. One first pertinent remark is that Theorem 10.5 is not a consequence of the previous proposition 10.4 as pointed out by A. Grigor'yan in [7, p. 180-181]. Indeed, he shows by a nice example, that 10.5 is not equivalent to parabolicity of the model space, so, although 10.6 does imply parabolicity of the model space, we cannot conclude parabolicity of $P^{m}$ via Proposition 10.4 .

As a corollary to Theorem 10.5 we get Theorem $\mathrm{C}$ as follows:

Proof of Theorem $C$. The strong conditions for the theorem as stated in the introduction, i.e. $h(r)=C$ and $w(r)=Q_{b}(r), b \leq 0$, still implies that the model space in the comparison constellation, $C_{Q_{b}, g, C}^{m}$, is $Q_{b}$-balanced from below. Furthermore, since we have that $1 \geq$ $g(r)>0$, the 'stretching' satisfies $s(r) \rightarrow \infty$ when $r \rightarrow \infty$. We have 
then according to Proposition 5.13, (5.18):

$$
\begin{aligned}
& \int^{\infty} \frac{1}{\operatorname{Vol}\left(\partial B_{s}^{W}\right)} d s \\
& =\int^{\infty} \frac{1}{W^{m-1}(s(r))}\left(\frac{d s}{d r}\right) d r \\
& =\int^{\infty} \frac{1}{\Lambda(r) g(r)} d r \\
& =\int^{\infty}\left(\frac{T}{Q_{b}(r)} \exp \left(-\int_{r}^{1} \frac{m}{g^{2}(t)}\left(\eta_{Q_{b}}(t)-C\right) d t\right)\right)^{-1} d r
\end{aligned}
$$

where $T$ is the fixed constant defined and found in Proposition 5.13. In this particular case the condition (10.6) therefore corresponds to (2.7) in Theorem C, which is thence proved.

The intrinsic versions of these results (implying formally that $h(r)=$ 0 and $g(r)=1$ for all $r$ ) are well known and established by Ahlfors, Nevanlinna, Karp, Varopoulos, Lyons and Sullivan, and Grigor'yan, see [7, Theorem 7.3 and Theorem 7.5]. For warped product model space manifolds, the reciprocal boundary-volume integral condition is also necessary for parabolicity, but in general none of the conditions are necessary.

\section{Capacity Analysis}

Given the extrinsic balls with radii $\rho<R, D_{\rho}$ and $D_{R}$, the annulus $A_{\rho, R}$ is defined as

$$
A_{\rho, R}=D_{R} \sim D_{\rho}
$$

The unit normal vector field on the boundary of this annulus $\partial A_{\rho, R}=$ $\partial D_{\rho} \cup \partial D_{R}$ is denoted by $\nu$ and defined by the following normalized $P$-gradient of the distance function restricted to $\partial D_{\rho}$ and $\partial D_{R}$, respectively:

$$
\nu=\nabla^{P} r(x) /\left\|\nabla^{P} r(x)\right\|, \quad x \in \partial A_{\rho, R} .
$$

We apply the previously mentioned result by Greene and $\mathrm{Wu},[6]$, with a lower radial curvature bound again, but now we consider radial functions with $f^{\prime}(r) \geq 0$ which will change the inequality in the Laplace comparison, equation (5.17).

Theorem 11.1. Let $\left\{N^{n}, P^{m}, C_{w, g, h}^{m}\right\}$ denote an isoperimetric comparison constellation which is $w$-balanced from below on $[0, \infty[$. Then

$$
\operatorname{Cap}\left(A_{\rho, R}\right) \leq\left(\int_{s(\rho)}^{s(R)} \frac{1}{W^{m-1}(t)} d t\right)^{-1} .
$$


Proof. We consider again the second order differential operator L but now we look for the smooth solution $\xi(r)$ to the following DirichletLaplace problem on the interval $[\rho, R], \rho>0$ :

$$
\begin{aligned}
\mathrm{L} \xi(r) & =0 \quad \text { on }[\rho, R], \\
\xi(\rho) & =0 \quad, \quad \xi(R)=1 .
\end{aligned}
$$

The solution is again constructed via the function $\Lambda(r)$ defined in equation (5.18):

$$
\xi^{\prime}(r)=\Xi(r)=\frac{1}{g(r) \Lambda(r)}\left(\int_{\rho}^{R} \frac{1}{g(t) \Lambda(t)} d t\right)^{-1},
$$

Then we have

$$
\xi(r)=\left(\int_{\rho}^{r} \frac{1}{g(t) \Lambda(t)} d t\right)\left(\int_{\rho}^{R} \frac{1}{g(t) \Lambda(t)} d t\right)^{-1} .
$$

Applying the Laplace inequality (5.17) on the radial functions $\xi(r)$ now with a non-negative derivative - transplanted into $P^{m}$ in $N^{n}$ now gives the following comparison inequality, using the assumptions stated in the theorem:

$$
\begin{aligned}
\Delta^{P} \xi(r(x)) \leq & \left(\xi^{\prime \prime}(r(x))-\xi^{\prime}(r(x)) \eta_{w}(r(x))\right) g^{2}(r(x)) \\
& +m \xi^{\prime}(r(x))\left(\eta_{w}(r(x))-h(r(x))\right) \\
= & \mathrm{L} \xi(r(x)) \\
= & 0 \\
= & \Delta^{P} v(x)
\end{aligned}
$$

where $v(x)$ is the Laplace potential function for the extrinsic annulus $A_{\rho, R}=D_{R}-D_{\rho}$, setting $v_{\partial D_{\rho}}=0$ and $v_{\partial D_{R}}=1$.

For this inequality to hold we need that

$$
\xi^{\prime \prime}(r)-\xi^{\prime}(r) \eta_{w}(r) \leq 0 \quad .
$$

This follows from the Laplace equation itself together with the consequence (5.33) of the balance condition that $h(r) \leq \eta_{w}(r)$ :

$$
\left(\xi^{\prime \prime}(r)-\xi^{\prime}(r) \eta_{w}(r)\right) g^{2}(r)=-m \xi^{\prime}(r)\left(\eta_{w}(r)-h(r)\right) \leq 0 .
$$

The maximum principle then applies again and gives:

$$
v(x) \leq \xi(r(x)), \text { for all } x \in A_{\rho, R} .
$$

This implies in particular that at $\partial D_{\rho}$ we have

$$
\Xi(\rho)=\left|\xi^{\prime}(\rho)\right| \geq\left\|\nabla^{P} v(x)_{\left.\right|_{\partial D_{\rho}}}\right\|
$$


Applying the divergence theorem and using Corollary 8.3, we get

$$
\begin{aligned}
\operatorname{Cap}\left(A_{\rho, R}\right) & =\int_{\partial D_{\rho}}\left\|\nabla^{P} v(x)\right\| d \nu \\
& \leq \Xi(\rho) \int_{\partial D_{\rho}}\left\|\nabla^{P} r\right\| d \nu \\
& \leq \Xi(\rho) g(\rho) \Lambda(\rho) \\
& =\left(\int_{\rho}^{R} \frac{1}{g(t) \Lambda(t)} d t\right)^{-1} \\
& =\left(\int_{s(\rho)}^{s(R)} \frac{1}{W^{m-1}(t)} d t\right)^{-1}
\end{aligned}
$$

which shows the general capacity bound (11.1).

We then finally have the following promised consequence alluded to in the previous section:

Corollary 11.2 (Theorem 10.5). Under the conditions of Theorem 11.1: If furthermore

$$
\int^{\infty} \frac{1}{W^{m-1}(t)} d t=\infty
$$

then $P^{m}$ is parabolic.

Proof. Referring to the above capacity inequality (11.1), we see that the capacity is forced to 0 in the limit $s \rightarrow \infty$ because of the condition (11.11). According to the Kelvin-Nevanlinna-Royden criterion (b) of the introduction, see subsection 1.1. this corresponds to parabolicity of the submanifold.

\section{REFERENCES}

[1] Chavel, I. Eigenvalues in Riemannian geometry, vol. 115 of Pure and Applied Mathematics. Academic Press Inc., Orlando, FL, 1984. Including a chapter by Burton Randol, With an appendix by Jozef Dodziuk.

[2] Do Carmo, M. P., And Warner, F. W. Rigidity and convexity of hypersurfaces in spheres. J. Differential Geometry 4 (1970), 133-144.

[3] Fernández, J. L. On the existence of Green's function in Riemannian manifolds. Proc. Amer. Math. Soc. 96, 2 (1986), 284-286.

[4] Gray, A., And Pinsky, M. A. The mean exit time from a small geodesic ball in a Riemannian manifold. Bull. Sci. Math. (2) 107, 4 (1983), 345-370.

[5] Gray, A., And VAnhecke, L. Riemannian geometry as determined by the volumes of small geodesic balls. Acta Math. 142, 3-4 (1979), 157-198.

[6] Greene, R. E., And Wu, H. Function theory on manifolds which possess a pole, vol. 699 of Lecture Notes in Mathematics. Springer, Berlin, 1979.

[7] GRIGOR'YyAn, A. Analytic and geometric background of recurrence and nonexplosion of the Brownian motion on Riemannian manifolds. Bull. Amer. Math. Soc. (N.S.) 36, 2 (1999), 135-249. 
[8] IChinarA, K. Curvature, geodesics and the Brownian motion on a Riemannian manifold. I. Recurrence properties. Nagoya Math. J. 87 (1982), 101-114.

[9] ICHIHARA, K. Curvature, geodesics and the Brownian motion on a Riemannian manifold. II. Explosion properties. Nagoya Math. J. 87 (1982), 115-125.

[10] Jorge, L., And Koutroufiotis, D. An estimate for the curvature of bounded submanifolds. Amer. J. Math. 103, 4 (1981), 711-725.

[11] Karp, L., And Pinsky, M. Mean exit time from an extrinsic ball. In From local times to global geometry, control and physics (Coventry, 1984/85), vol. 150 of Pitman Res. Notes Math. Ser. Longman Sci. Tech., Harlow, 1986, pp. 179186.

[12] KarP, L., AND Pinsky, M. Volume of a small extrinsic ball in a submanifold. Bull. London Math. Soc. 21, 1 (1989), 87-92.

[13] Kobayashi, S., and Nomizu, K. Foundations of differential geometry. Vol. I, II. A Wiley-Interscience Publication, 1963, 1969.

[14] Lyons, T., AND Sullivan, D. Function theory, random paths and covering spaces. J. Differential Geom. 19, 2 (1984), 299-323.

[15] Markvorsen, S. On the heat kernel comparison theorems for minimal submanifolds. Proc. Amer. Math. Soc. 97, 3 (1986), 479-482.

[16] Markvorsen, S. Distance geometric analysis on manifolds. In Global Riemannian geometry: curvature and topology, Adv. Courses Math. CRM Barcelona. Birkhäuser, Basel, 2003, pp. 1-54.

[17] Markvorsen, S., McGuinness, S., and Thomassen, C. Transient random walks on graphs and metric spaces with applications to hyperbolic surfaces. Proc. London Math. Soc. (3) 64, 1 (1992), 1-20.

[18] Markvorsen, S., And Palmer, V. Generalized isoperimetric inequalities for extrinsic balls in minimal submanifolds. J. Reine Angew. Math. 551 (2002), $101-121$.

[19] Markvorsen, S., And Palmer, V. Transience and capacity of minimal submanifolds. Geom. Funct. Anal. 13, 4 (2003), 915-933.

[20] Markvorsen, S., And Palmer, V. How to obtain transience from bounded radial mean curvature. Trans. Amer. Math. Soc. 357, 9 (2005), 3459-3479 (electronic).

[21] Markvorsen, S., And Palmer, V. Torsional rigidity of minimal submanifolds. Proc. London Math. Soc. (3) 93, 1 (2006), 253-272.

[22] Milnor, J. On deciding whether a surface is parabolic or hyperbolic. Amer. Math. Monthly 84, 1 (1977), 43-46.

[23] O'NeILl, B. Semi-Riemannian geometry, vol. 103 of Pure and Applied Mathematics. Academic Press Inc. [Harcourt Brace Jovanovich Publishers], New York, 1983. With applications to relativity.

[24] Palmer, V. Mean exit time from convex hypersurfaces. Proc. Amer. Math. Soc. 126, 7 (1998), 2089-2094.

[25] PAlmer, V. Isoperimetric inequalities for extrinsic balls in minimal submanifolds and their applications. J. London Math. Soc. (2) 60, 2 (1999), 607-616.

[26] Sakai, T. Riemannian geometry, vol. 149 of Translations of Mathematical Monographs. American Mathematical Society, Providence, RI, 1996. Translated from the 1992 Japanese original by the author.

[27] SPIVAK, M. A comprehensive introduction to differential geometry. Vol. I $V$, second ed. Publish or Perish Inc., Wilmington, Del., 1979. 
Department of Mathematics, Technical University of Denmark.

E-mail address: S.Markvorsen@mat.dtu.dk

Departament de Matemàtiques, Universitat Jaume I, Castellon, Spain.

E-mail address: palmer@mat.uji.es 